\title{
On Drinfeld's universal formal group over the $p$-adic upper half plane
}

\author{
Jeremy Teitelbaum* \\ Department of Mathematics, University of Michigan, Ann Arbor, MI 48109, USA
}

\section{Introduction}

In his important paper "Coverings of p-adic symmetric regions" [Dr], Drinfeld showed that the $p$-adic upper half plane and its higher dimensional analogues serve as moduli spaces for certain rigidified formal groups with quaternionic multiplications. Given a formal group of the proper type, together with rigidifying data, over, say, a ring $R$ on which $p$ is nilpotent, Drinfeld constructs an $R$-valued point of the appropriate $p$-adic half space. If we draw an analogy between formal groups and abelian varieties, then Drinfeld's procedure is analogous to computing the period lattice of an abelian variety and obtaining thereby a point in a Siegel upper half space. In the case of abelian varieties, the inverse procedure is well known-given a period lattice, the abelian variety can be constructed immediately. The purpose of this paper is to supply the corresponding inverse procedure in the case of Drinfeld's formal groups, at least in a special case. More precisely, given a $W\left(\overline{\mathbf{F}}_{p}\right)$-valued point of the $p$-adic upper half plane over $\mathbf{Z}_{p}$, we construct the corresponding 2dimensional, height 4 quaternionic module over $W\left(\overrightarrow{\mathbf{F}}_{p}\right)$ with its rigidifying data.

We proceed in three stages. First, we discuss in detail Drinfeld's functorial description of points on the p-adic upper half plane. We supply a proof of his description, which he states without proof in [Dr]. We also obtain a rigid analytic interpretation of his description. Next, we construct the special fiber of the formal quaternionic module which corresponds to a point on the reduction mod $p$ of the $p$ adic upper half plane. Finally, we apply the technique of the universal extension, as described in [Haz], to obtain our desired module over $W\left(\overline{\mathbf{F}}_{p}\right)$. We supply the Dieudonne module and the logarithm for this module in Theorems 45 and 46.

\section{Notation}

Let $D$ be the quaternion division algebra over $\mathbf{Q}_{p}$, and let $\mathcal{O}_{D}$ be the maximal order in $D$. Choose $\Pi$ in $\mathcal{O}_{D}$ so that $\Pi^{2}=p$, and let

$$
A=\left\{a \in \mathcal{O}_{D}: \Pi a=\bar{a} \Pi\right\}
$$

\footnotetext{
* This research was partially supported by an NSF Postdoctoral Fellowship
} 
where $a \mapsto \bar{a}$ is the involution on $D . A$ is isomorphic to the quadratic unramified extension of $\mathbf{Z}_{p}$.

If $G$ is a commutative formal group over a $\mathbf{Z}_{(p)}$ algebra $R$, then we let $\mathbf{M}(G)$ denote the Cartier-Dieudonne module of $p$-typical curves for $G$. We let $W(R)$ denote the (1-prime) Witt vectors of $R$. In general, all of our notations and conventions for Witt vectors and Cartier-Dieudonne modules will follow Hazewinkel [Haz].

We let $\hat{\mathscr{H}}_{p}$ be the formal scheme constructed by Mumford [Mum], and generally referred to as the $p$-adic upper half plane over $\mathbf{Z}_{p}$. We let $\mathscr{H}_{p}$ denote the associated rigid analytic space over $\mathbf{Q}_{p}$. Thus the set of $L$-valued points of $\mathscr{H}_{p}$ is $L-\mathbf{Q}_{p}$ for extension fields $L$ of $\mathbf{Q}_{p}$.

The definition of a special formal $\mathcal{O}_{D}$ module is due to Drinfeld; we recall it here.

Definition 1. Let $B$ be $\mathbf{Z}_{\mathrm{p}}$-algebra. $A 2$ dimensional commutative formal group $G$ over $B$, together with an embedding

$$
l: \mathcal{O}_{D} \hookrightarrow \operatorname{End}(G)
$$

is called a formal $\mathscr{O}_{D}$ module. If, at each geometric point $P$ of $\operatorname{Spec} B / p B$, the representation of $\mathcal{O}_{D} / \Pi \mathcal{O}_{D}$ on the tangent space of $G \times \kappa_{P}$ is the sum of two distinct characters of $\mathscr{O}_{D} / \Pi \mathcal{O}_{D}$, then $G$ is called special.

We will abbreviate the phrase " $G$ is a special, formal $\mathscr{O}_{D}$ module" by saying that $G$ is an SFD-module.

If $G$ over $B$ is an SFD-module, then the endomorphisms of $G$ endow the Dieudonne module of $G$ with additional structure. To make this explicit, label the two embeddings

$$
\sigma_{0}, \sigma_{1}: \mathcal{O}_{D} / I \mathcal{O}_{D} \rightarrow \overline{\mathbf{F}}_{p}
$$

These embeddings determine two embeddings $A \rightarrow W\left(\overline{\mathbf{F}}_{p}\right)$; we will abuse notation and denote the lifted embeddings with the same letter. Then $\mathbf{M}(G)$ carries a $\mathbf{Z} / 2 \mathbf{Z}$ grading, defined by

$$
\mathbf{M}(G)^{i}=\left\{x \in \mathbf{M}(G): z(a) \cdot x=\sigma_{i}(a) x a \in A\right\} .
$$

The operations $\mathbf{F}$ and $\mathbf{V}$, and the endomorphism $\Pi$, have degree 1 relative to this grading.

An isogeny between two formal groups $G$ and $H$ over $B$ is a homomorphism $\rho: G \rightarrow H$ having finite kernel. If $B$ is a finite field of characteristic $p$, and $\rho$ is an isogeny from $G$ to $H$, then the kernel $N$ of $\rho$ is a finite connected group scheme over $B$. The rank of $N$ is $p^{h}$ for some integer $h$; we call this integer $h$ the height of $\rho$.

Following Drinfeld, we say that an element $\rho$ of $\operatorname{Hom}(G, H) \otimes \mathbf{Q}_{p}$ is a quasiisogeny if $\rho$ has an inverse in $\operatorname{Hom}(H, G) \otimes \mathbf{Q}_{p}$. The height function extends to $\operatorname{Hom}(G, H) \otimes \mathbf{Q}_{p}$ in a natural way.

\section{The p-adic upper half plane revisited}

Viewed from a naive perspective, the upper half plane $\hat{\mathscr{H}}_{p}$ is the complement of the rational points of $\mathbf{P}^{1}$. In order to prove his theorem on moduli (Theorem 28 below) Drinfeld describes the points of $\mathscr{\mathscr { H }}_{p}$ in schemes $S$ on which $p$ is nilpotent functorially, 
in terms of certain data on $S$. We will now embark on something of a digression in order to describe this data and to make explicit how it is used to construct a morphism $S \rightarrow \hat{\mathscr{H}}_{p}$. Then, we will construct universal data on $\hat{\mathscr{H}}_{p}$ so that a map $S \rightarrow \hat{\mathscr{H}}_{p}$ yields appropriate data on $S$ by pull-back. A major tool in our study will be an interpretation of Drinfeld's functorial description of the $S$-valued points of $\hat{\mathscr{H}}_{p}$ from a rigid analytic perspective.

\section{Preliminaries}

Let us begin by fixing some fundamentals regarding $\mathscr{H}_{p}$ and $\hat{\mathscr{H}}_{p}$. In particular, there is a well-known relationship between the tree $\mathscr{T}$ of $G L_{2}\left(\mathbf{Q}_{p}\right)$ and $\mathscr{H}_{p}$. (See $[\mathrm{Mum}]$ or [Man]) We will realize this relationship in the following way. Fix a coordinate function $z$ on $\mathscr{H}_{p}$. There is a canonical reduction map

$$
r_{\mathscr{T}}: \mathscr{H}_{\boldsymbol{p}} \rightarrow \mathscr{F}
$$

such that, if $\tau \in G L_{2}\left(\mathbf{Q}_{p}\right)$, and $v(\tau)$ is the corresponding vertex, then

$$
r_{\mathscr{T}}^{-1}(v(\tau))=\left\{P \in \mathbf{P}^{1}:|\tau z(P)|=1\right\}-\bigcup_{i=0}^{p-1} \tau^{-1} B_{1}(i)
$$

where $B_{r}(x)$ is the open ball of radius $r$ centered at $x . G L_{2}\left(\mathbf{Q}_{p}\right)$ acts on $\mathscr{T}$ by the rule

$$
\tau_{1} \cdot v\left(\tau_{2}\right)=v\left(\tau_{2} \tau_{1}^{-1}\right),
$$

and this action commutes with the reduction map and with the action of $G L_{2}\left(\mathbf{Q}_{p}\right)$ on $\mathscr{H}_{p}$ through linear fractional transformations in $z$.

Definition 2. Let $\Gamma^{0}(p)$ be the subgroup of $S L_{2}\left(Z_{p}\right)$ consisting of matrices with upper right entry divisible by $p$.

Lemma 1. The edges of $\mathscr{T}$ are in one-to-one correspondence with the coset space $\Gamma^{0}(p) \backslash S L_{2}\left(\mathbf{Q}_{p}\right)$.

Proof. There is an $S L_{2}\left(\mathbf{Q}_{p}\right)$ invariant orientation on the edges of $\mathscr{T}$, and $S L_{2}\left(\mathbf{Q}_{p}\right)$ permutes the edges of $\mathscr{T}$ transitively within this orientation. The group $\Gamma^{0}(p)$ is the stabilizer of the edge joining the vertices $v(1)$ and $v(m)$, where

$$
m=\left(\begin{array}{ll}
0 & p \\
1 & 0
\end{array}\right) . \square
$$

If $\tau \in S L_{2}\left(\mathbf{Q}_{p}\right)$, we will use the notation $e(\tau)$ to denote the edge in $\mathscr{T}$ corresponding to $\tau$. Let $\bar{e}(\tau)$ denote the "dumbell' consisting of the edge $e(\tau)$ together with its two terminal vertices. We set $U(\tau)=r_{\mathscr{F}}^{-1}(\bar{e}(\tau))$. Each $U(\tau)$ is an affinoid subdomain of $\mathscr{H}_{p}$, and together they form an admissible covering of $\mathscr{H}_{p}$. For the sake of concreteness, we point out that

$$
U(1)=\left\{P \in \mathbf{P}^{1}:|z(P)| \leqq 1\right\}-\bigcup_{i=1}^{p-1}\left(B_{1}(i) \cup B_{1 / p}(p i)\right)-B_{1 / p}(0)
$$

while $U(\tau)=\tau^{-1} U(1)$ for $\tau \in S L_{2}\left(\mathbf{Q}_{p}\right)$. 
We also point out that the correspondence between edges $e$ in the tree $\mathscr{T}$ and cosets $\Gamma^{0}(p) \backslash S L_{2}\left(\mathbf{Q}_{p}\right)$ implies the following relations:

Lemma 2. The definition of the $U(\tau)$ yield the following relations:

1. $U\left(\tau_{1}\right)=U\left(\tau_{2}\right)$ if and only if $\tau_{1} \tau_{2}^{-1} \in \Gamma^{0}(p)$.

2. If $\tau_{1} \tau_{2}^{-1} \in S L_{2}\left(\mathbf{Z}_{p}\right)$ but $\tau_{1} \tau_{2}^{-1} \notin m^{-1} S L_{2}\left(\mathbf{Z}_{p}\right) m$, then $v\left(\tau_{1}\right)=v\left(\tau_{2}\right)$ and $U\left(\tau_{1}\right) \cap U\left(\tau_{2}\right)=r_{g^{-}}^{-1}\left(v\left(\tau_{1}\right)\right)$.

3. If $\tau_{1} \tau_{2}^{-1} \in m^{-1} S L_{2}\left(\mathbf{Z}_{p}\right) m$, but $\tau_{1} \tau_{2}^{-1} \notin S L_{2}\left(\mathbf{Z}_{p}\right)$, then $v\left(m \tau_{1}\right)=v\left(m \tau_{2}\right)$ and $U\left(\tau_{1}\right) \cap U\left(\tau_{2}\right)=r_{\mathscr{T}}^{-1}\left(v\left(m \tau_{1}\right)\right)$.

4. If none of the above conditions hold, then $U\left(\tau_{1}\right) \cap U\left(\tau_{2}\right)=\varnothing$.

\section{The formal scheme $\hat{\mathscr{H}}_{p}$ - the naive construction}

Now we give a construction of $\hat{\mathscr{H}}_{p}$ which will supply us with a naive description of the $S$-valued points in $\hat{\mathscr{H}}_{p}$. This construction is derived from Bosch's work $[\mathrm{B}]$ on passage from rigid spaces to formal schemes.

Associated with the covering $\{U(\tau)\}$ of $\mathscr{H}_{p}$ is a reduction $\overline{\mathscr{H}_{p}}$, together with a map $r: \mathscr{H}_{p} \rightarrow \overline{\mathscr{H}}_{p} . \overline{\mathscr{H}}_{p}$ is a scheme over $\mathbf{F}_{p}$ consisting of rational curves meeting in ordinary double points with intersection graph $\mathscr{T}$. The sets $r\left(U\left(\tau_{1}\right) \cap U\left(\tau_{2}\right)\right)$ are Zariski open in the reduction $\overline{\mathscr{H}}_{p}$. Following [B], we may derive a formal scheme $\mathscr{\mathscr { H }}_{p}$ from $\mathscr{H}_{p}$. The underlying topological space of $\hat{\mathscr{H}}_{p}$ is the same as that of $\overline{\mathscr{H}}_{p}$. If, however, $\bar{U}$ is an open set in $\overline{\mathscr{H}_{p}}$, then

$$
\mathcal{O}_{\hat{\mathscr{H}}_{p}}(\bar{U})=\left\{f \in \mathcal{O}_{\mathscr{*}_{p}}\left(r^{-1}(\bar{U})\right):|f|_{r}^{-1}(\bar{U}) \leqq 1\right\} \text {. }
$$

In a sense, $\hat{\mathscr{H}}_{p}$ is a model for $\mathscr{H}_{p}$ over $\mathbf{Z}_{p}$.

If $S$ is a scheme over $\mathbf{Z}_{p}$ on which $p$ is nilpotent, we would like to find a description of the $S$ valued points of $\widehat{\mathscr{H}}_{p}$. To this end, let us make the construction of $\mathscr{H}_{p}$ absolutely explicit. As $\tau$ runs through $S L_{2}\left(Q_{p}\right)$, let

and

$$
R(\tau)=\mathbf{Z}\left[z_{\tau}, w_{\tau}, \frac{1}{1-z_{\tau}^{p-1}}, \frac{1}{1-w_{\tau}^{p-1}}\right] /\left(z_{\tau} w_{\tau}-p\right)
$$

$$
\hat{R}(\tau)=\lim R / p^{n} R
$$

Then it follows from the definitions, and in particular from (1), that

$$
\mathcal{O}_{\hat{\mathscr{H}}_{p}}(\bar{U}(\tau)) \cong \hat{R}(\tau)
$$

Lemma 3. The gluing maps between the various $\bar{U}(\tau)$ are determined by the following cases:

Case 1. $\tau_{1} \tau_{2}^{-1} \in \Gamma^{0}(p)$. Then the map

$$
\begin{aligned}
& \hat{R}\left(\tau_{1}\right) \rightarrow \hat{R}\left(\tau_{2}\right) \\
& z_{\tau_{1}} \mapsto \tau_{1} \tau_{2}^{-1} z_{\tau_{2}} \\
& w_{\tau_{1}} \mapsto m^{-1} \tau_{1} \tau_{2}^{-1} m w_{\tau_{2}}
\end{aligned}
$$

defines an isomorphism $\hat{R}\left(\tau_{1}\right) \rightarrow \hat{R}\left(\tau_{2}\right)$. 
Case 2. $\tau_{1} \tau_{2}^{-1} \in S L_{2}\left(\mathbf{Z}_{p}\right)$ but $\tau_{1} \tau_{2}^{-1} \notin m^{-1} S L_{2}\left(\mathbf{Z}_{p}\right) m$. Then the map

$$
\begin{gathered}
\hat{R}\left(\tau_{1}\right)\left[\frac{1}{z_{\tau_{1}}}\right] \rightarrow \hat{R}\left(\tau_{2}\right)\left[\frac{1}{z_{\tau_{2}}}\right] \\
z_{\tau_{1}} \mapsto \tau_{1} \tau_{2}^{-1} z_{\tau_{2}}
\end{gathered}
$$

glues the open sets where $z_{\tau_{1}}$ and $z_{\tau_{2}}$ are units.

Case 3. $\tau_{1} \tau_{2}^{-1} \in m^{-1} S L_{2}\left(\mathbf{Z}_{p}\right) m$ but $\tau_{1} \tau_{2}^{-1} \notin S L_{2}\left(\mathbf{Z}_{p}\right)$. Then the map

$$
\begin{aligned}
& \hat{R}\left(\tau_{1}\right)\left[\frac{1}{w_{\tau_{1}}}\right] \rightarrow \hat{R}\left(\tau_{2}\right)\left[\frac{1}{w_{\tau_{2}}}\right] \\
& w_{\tau_{1}} \mapsto m^{-1} \tau_{1} \tau_{2}^{-1} m w_{\tau_{2}}
\end{aligned}
$$

glues the open sets where $w_{\tau_{1}}$ and $w_{\tau_{2}}$ are units.

Proof. All of this follows from consideration of the tree $\mathscr{T}$; we refer the reader to [Mum] or [Man] for details.

This discussion enables us to prove the following lemma.

Lemma 4. Let $S$ be a $\mathbf{Z}_{p}$ scheme on which $p$ is nilpotent. Then to specify a morphism $\Psi: S \rightarrow \hat{\mathscr{H}}_{p}$ is equivalent to giving the following data:

1. A covering $\{V(\tau)\}$ of $S$ by open sets, indexed by elements of $S L_{2}\left(\mathbf{Q}_{p}\right)$.

2. A pair of functions $z_{\tau}$ and $w_{\tau}$ on $V(\tau)$ which satisfy $z_{\tau} w_{\tau}=p$ and such that $1-z_{\tau}^{p-1}$ and $1-w_{\tau}^{p-1}$ are units on $V(\tau)$.

The open sets $V(\tau)$ with their functions $z_{\mathfrak{r}}$ and $w_{\tau}$ must satisfy the following conditions:

Case 1. If $\tau_{1} \tau_{2}^{-1} \in \Gamma^{0}(p)$, then $V\left(\tau_{1}\right)=V\left(\tau_{2}\right)$ and

$$
\begin{aligned}
& z_{\tau_{1}}=\tau_{1} \tau_{2}^{-1} z_{\mathfrak{t}_{2}} \\
& w_{\tau_{1}}=m^{-1} \tau_{1} \tau_{2}^{-1} m w_{\tau_{2}} .
\end{aligned}
$$

Case 2. If $\tau_{1} \tau_{2}^{-1} \in S L_{2}\left(\mathbf{Z}_{p}\right)$ but $\tau_{1} \tau_{2}^{-1} \notin m^{-1} S L_{2}\left(\mathbf{Z}_{p}\right) m$, then

$$
V\left(\tau_{1}\right) \cap V\left(\tau_{2}\right)=V\left(\tau_{1}\right)\left[z_{\tau_{1}}^{-1}\right]=V\left(\tau_{2}\right)\left[z_{\tau_{2}}^{-1}\right]
$$

and on this open set

$$
z_{\tau_{1}}=\tau_{1} \tau_{2}^{-1} z_{\tau_{2}}
$$

Case 3. If $\tau_{1} \tau_{2}^{-1} \in m^{-1} S L_{2}\left(\mathbf{Z}_{p}\right) m$ but $\tau_{1} \tau_{2}^{-1} \notin S L_{2}\left(\mathbf{Z}_{p}\right)$ then

$$
V\left(\tau_{1}\right) \cap V\left(\tau_{2}\right)=V\left(\tau_{1}\right)\left[w_{\tau_{1}}^{-1}\right]=V\left(\tau_{2}\right)\left[w_{\tau_{2}}^{-1}\right]
$$

and on this open set

$$
w_{\tau_{1}}=m^{-1} \tau_{1} \tau_{2}^{-1} m w_{\tau_{2}} .
$$

Proof. Specifying $z_{\tau}$ and $w_{\tau}$ on $V(\tau)$ determines a map $\psi_{\tau}: V(\tau) \rightarrow \bar{U}(\tau)$. The additional conditions guarantee that the $\psi_{\tau}$ glue to give a map $\Psi: S \rightarrow \hat{\mathscr{H}}_{p}$. 
Drinfeld's description of $\hat{\mathscr{H}}_{p}$

Armed with the above naive description of the points of $\hat{\mathscr{H}}_{p}$, we now consider Drinfeld's description, given in [Dr, p. 109]. He claims that points of $\hat{\mathscr{H}}_{p}$ in formal schemes $S$ on which $p$ is nilpotent are determined by certain data on $S$. Here, we will recall the definition of his data, and show how to deduce from it a map from $S$ to $\hat{\mathscr{H}}_{p}$. Later, we construct universal data on $\widehat{\mathscr{H}}_{p}$ to show that any map from $S$ to $\widehat{\mathscr{H}}_{p}$ induces Drinfeld's data on $S$.

If $B$ is a ring, Drinfeld denotes by $B[\Pi]$ the ring $B[\Pi] /\left(\Pi^{2}-p\right)$, and attaches a grading to $B[\Pi]$ by letting $\operatorname{deg}(b)=0$ for $b \in B$ and $\operatorname{deg}(\Pi)=1$. Suppose $S$ is a $\mathbf{Z}_{p}$ scheme on which $p$ is nilpotent. Then an $S$-valued point of $\hat{\mathscr{H}}_{p}$ consists of the following data:

(1) A sheaf of flat, $\mathbf{Z} / 2 \mathbf{Z}$-graded $\mathbf{Z}_{p}[\Pi]$ modules $\eta$ on $S$. (note that $\eta$ is not a quasicoherent sheaf, but only a sheaf of abelian groups with added structure.)

(2) A Z/2Z-graded sheaf $T$ of $\mathcal{O}_{S}[\Pi]$ modules whose homogeneous components are invertible.

(3) A homogeneous, degree zero map $u: \eta \rightarrow T$ such that $u \otimes 1: \eta \otimes \mathcal{O}_{S} \rightarrow T$ is surjective.

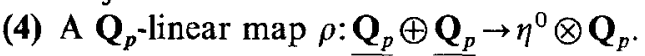

All of this data must be compatible, in the sense that it must satisfy the following conditions:

(C1) Let $S_{i} \subset S$ be the zeros of the morphism $\Pi: T^{i} \rightarrow T^{i+1}$; then $\left.\eta^{i}\right|_{S_{1}}$ is a constant sheaf with fiber $\mathbf{Z}_{p} \oplus \mathbf{Z}_{p}$.

(C2) For all points $s$ of $S$, the map

$$
\bar{u}: \eta_{s} / \Pi \eta_{s} \rightarrow\left(T_{s} / \Pi T_{s}\right) \otimes \kappa_{s}
$$

is injective, where $\kappa_{s}$ is the residue field of $s$.

(C3)

$$
\left.\Lambda^{2}\left(\eta^{i}\right)\right|_{s_{i}}=\left.p^{-i} \Lambda^{2}\left(\Pi^{i} \rho\left(\underline{\mathbf{Z}_{p}} \oplus \underline{\mathbf{Z}_{p}}\right)\right)\right|_{s_{i}} .
$$

Let us now show how Drinfeld's data give rise to maps $S \rightarrow \hat{\mathscr{H}}_{p}$. We will construct a covering $\{V(\tau)\}$ of $S$ together with functions $z_{\tau}$ and $w_{\tau}$ meeting the requirements of lemma 4 .

Let $x_{\mathbf{0}}$ and $x_{1}$ be the standard generators of the lattice $\mathbf{Z}_{p} \oplus \mathbf{Z}_{p} \subset \mathbf{Q}_{p} \oplus \mathbf{Q}_{p}$. For any element $\tau \in S L_{2}\left(\mathbf{Q}_{p}\right)$, define

Let us also set

$$
\left(\begin{array}{l}
x_{0}(\tau) \\
x_{1}(\tau)
\end{array}\right)=\left(\begin{array}{ll}
a & b \\
c & d
\end{array}\right)\left(\begin{array}{l}
x_{0} \\
x_{1}
\end{array}\right)
$$

and

$$
L_{0}(\tau)=\mathbf{Z}_{p} x_{0}(\tau)+\mathbf{Z}_{p} x_{1}(\tau)
$$

$$
L_{1}(\tau)=\mathbf{Z}_{p} p x_{0}(\tau)+\mathbf{Z}_{p} x_{1}(\tau)
$$

Definition 3. For elements $\tau \in S L_{2}\left(\mathbf{Q}_{p}\right)$, let $V(\tau) \subset S$ be the subset

$$
V(\tau)=\left\{P \in S: \eta_{P}^{0} \supseteq \rho\left(L_{0}(\tau)\right) \text { and } \Pi \Pi \eta_{P}^{1} \supseteq \rho\left(L_{1}(\tau)\right)\right\} .
$$


Lemma 5. The sets $V(\tau)$ are open and cover $S$.

Proof. Let $U$ be a connected open set containing $P$. Then the existence of the isomorphism $\rho$ forces the restriction maps $\rho_{U V}: \eta^{\circ}(U) \rightarrow \eta^{0}(V)$ to be injective for any $V \subseteq U$. Therefore if $P$ is any point such that $\left.\eta^{0}\right|_{P} \supseteq \rho\left(L_{0}(\tau)\right)$, there is an open set $U(P)$ containing $P$ such that $\left.\eta^{0}\right|_{U(P)}$ contains $\rho\left(L_{0}(\tau)\right)$. It follows that the set

$$
V_{0}(\tau)=\left\{P \in S: \eta_{P}^{0} \supseteq \rho\left(L_{0}(\tau)\right)\right\}
$$

is open. A similar argument shows that

$$
V_{1}(\tau)=\left\{P \in S: \Pi \eta_{P}^{1} \supseteq \rho\left(L_{1}(\tau)\right)\right\}
$$

is also open, and therefore $V(\tau)=V_{1}(\tau) \cap V_{0}(\tau)$ is open.

Now we must show that the $V(\tau)$ cover $S$. If $P$ is any point of $S$, then either $P \in S_{0}$ or $P \in S_{1}$. In the first case, $\left.\eta^{0}\right|_{P}=\rho\left(L_{0}(\tau)\right)$ for some $\tau$ by condition (C3). This $\tau$ may be chosen so that $\left.\Pi \eta^{1}\right|_{P}$, which is a sublattice of $\left.\eta^{0}\right|_{P}$ of index at most $p$, contains $\rho\left(L_{1}(\tau)\right)$. It follows that $P \in V(\tau)$ for some $\tau$. A similar argument using (C3) shows that the $V(\tau)$ cover $S_{1}$ as well.

Definition 4. Let $S_{i}(\tau)=S_{i} \cap V(\tau)$ for $i=0,1$.

Lemma 6. The following equations are consequences of the compatibility conditions, when the sets in question are non-empty.

1. On the open $S_{0}(\tau)-S_{1}(\tau)$,

$$
\eta^{0}=\Pi \eta^{1}=\rho\left(L_{0}(\tau)\right) \text {. }
$$

2. On the open set $S_{1}(\tau)-S_{0}(\tau)$,

$$
\Pi \eta^{1}=\rho\left(L_{1}(\tau)\right)
$$

and

$$
\eta^{0}=\rho\left((1 / p) L_{1}(\tau)\right)
$$

3. If $P \in S_{0}(\tau) \cap S_{1}(\tau)$,

$$
\dot{\eta}_{P}^{0}=\rho\left(L_{0}(\tau)\right)
$$

and

$$
\Pi \eta_{P}^{1}=\rho\left(L_{1}(\tau)\right)
$$

Proof. On $S_{0}(\tau)-S_{1}(\tau)$, the sheaf $T^{0} / \Pi T^{1}$ is zero. Therefore, by (C2), we know that $\eta^{0} / \Pi \eta^{1}=0$ on this set. It follows that $\eta^{0}=\Pi \eta^{1}$ on $S_{0}(\tau)-S_{1}(\tau)$. By (C1), $\eta^{0}$ is constant. By the definition of $V(\tau), \eta^{0} \supseteq \rho\left(L_{0}(\tau)\right)$ on this set. Finally, by (C3), we know that $\Lambda^{2} \eta^{0}=\Lambda^{2} \rho\left(L_{0}\right)$. We conclude that $\eta^{0}=\rho\left(L_{0}(\tau)\right)$. On $S_{1}-S_{0}$, we know that $T^{1} / \Pi T^{0}=0$, and therefore by $(\mathrm{C} 2)$ that $\eta^{1}=\Pi \eta^{0}$. This means that $\Pi \eta^{1}=p \eta^{0}$. It then follows from (C1) and (C3) that $\Pi \eta^{1}=\rho\left(L_{1}(\tau)\right)$ and therefore that $\eta^{0}=(1 / p) \rho\left(L_{1}(\tau)\right)$. Finally, if $P \in S_{0} \cap S_{1}$, we know that neither $T^{0} / \Pi T^{1}$ nor $T^{1} / \Pi T^{0}$ are zero. It follows that each of $\eta_{P}^{0} / \Pi \eta_{P}^{1}$ and $\eta_{P}^{1} / \Pi \eta_{P}^{0}$ are one dimensional over $\mathbf{F}_{p}$. Using (C1) and (C3) as before, we see that $\eta_{P}^{\circ}=\rho\left(L_{0}(\tau)\right)$ and $\Pi \eta_{P}^{1}=\rho\left(L_{1}(\tau)\right)$. 
Corollary 7. There exist sections $y_{0}(\tau)$ and $y_{1}(\tau)$ in $\eta^{1}(V(\tau))$ such that $\Pi y_{0}(\tau)=\rho\left(x_{0}\right)$ and $\Pi y_{1}(\tau)=\rho\left(p x_{1}(\tau)\right)$.

Proof. Inspection of the above results show that $\rho\left(x_{0}(\tau)\right)$ and $\rho\left(p x_{1}(\tau)\right)$ belong to $\Pi \eta^{1}$ on all of $V(\tau)$.

Lemma 8. Let $\lambda \in \mathbf{Z}_{p}$ and set

$$
t_{0}(\tau, \lambda)=u\left(\rho\left(x_{1}(\tau)-\lambda x_{0}(\tau)\right)\right) \in T^{0}(V(\tau))
$$

Then $t_{0}(\tau, \lambda)$ has no zeroes on $V(\tau)$. Let $y_{0}(\tau)$ and $y_{1}(\tau)$ be the sections defined in the corollary, and let

$$
t_{1}(\tau, \lambda)=u\left(y_{0}(\tau)-\lambda y_{1}(\tau)\right) \in T^{1}(V(\tau))
$$

Then $t_{1}(\tau, \lambda)$ has no zeroes on $V(\tau)$.

Proof. The element $x_{1}(\tau)-\lambda x_{0}(\tau)$ does not belong to $\Pi \eta^{1}$ anywhere on $S_{1}(\tau)$. It follows from (C2) that $t_{0}(\tau, \lambda)$ is not zero in $T^{0} / \Pi T^{1} \otimes \kappa_{P}$ for any $P \in S_{1}(\tau)$. On $S_{0}(\tau)-S_{1}(\tau)$, we know that $T^{0}=\Pi T^{1}$, and that $\rho\left(x_{1}(\tau)-\lambda x_{0}(\tau)\right)=\Pi w$ for some $w \in \eta^{1}(V(\tau))$. Since $\eta^{1} / \Pi \eta^{0}$ injects into $T^{1} / \Pi T_{0}$, we conclude that $u(w)$ does not vanish on $S_{0}(\tau)-S_{1}(\tau)$ and therefore neither does $t_{0}(\tau, \lambda)=\Pi u(w)$. Since $S_{0}(\tau)$ and $S_{1}(\tau)$ cover $V(\tau)$, we conclude that the section $t_{0}(\tau, \lambda)$ never vanishes. A parallel argument shows that the same is true for $t_{1}(\tau, \lambda)$.

Definition 5. If $V(\tau)$ is non-empty, define a function $z_{\tau}$ on $V(\tau)$ by the formula

$$
z_{\tau}=u\left(\rho\left(x_{0}(\tau)\right)\right) / t_{0}(\tau, 0)
$$

and a function $w_{\tau}$ on $V(\tau)$ by the formula

$$
w_{\tau}=u\left(y_{1}(\tau)\right) / t_{1}(\tau, 0)
$$

Lemma 9. The functions $z_{\tau}$ and $w_{\tau}$ satisfy $z_{\tau} w_{\tau}=p$. The functions $1-z_{\tau}^{p-1}$ and $1-w_{\tau}^{p-1}$ are units on $V(\tau)$,

Proof. The relation $z_{\tau} w_{\imath}=p$ is a consequence of the relations

$$
\begin{aligned}
& \Pi \rho\left(x_{0}(\tau)\right)=p y_{0}(\tau) \\
& \Pi \rho\left(x_{1}(\tau)\right)=y_{1}(\tau)
\end{aligned}
$$

and the fact that the map $u$ commutes with $\Pi$. The fact that $1-z_{\tau}^{p-1}$ and $1-w_{\tau}^{p-1}$ are units follows from lemma 8 , since

$$
1-\lambda z_{\tau}=t_{0}(\tau, \lambda) / t_{0}(\tau, 0) \neq 0
$$

for all $\lambda \in \mathbf{Z}_{p}$.

Lemma 10. $V(\tau)\left[z_{\tau}^{-1}\right]=S_{0}(\tau)-S_{1}(\tau)$ and $V(\tau)\left[w_{\tau}^{-1}\right]=S_{1}(\tau)-S_{0}(\tau)$. The closed subscheme $S_{0}(\tau) \cap S_{1}(\tau)$ is defined by the ideal $\left(z_{\tau}, w_{\tau}\right)$.

Proof. By lemma $8, T^{0}$ is generated over $V(\tau)$ by the section $t_{0}(\tau, 0)$ and $T^{1}$ is 
generated by $t_{1}(\tau, 0)$. By the definitions, $\Pi t_{0}(\tau, 0)=w_{\tau} t_{1}(\tau, 0)$ and $\Pi t_{1}(\tau, 0)=$ $z_{\tau} t_{0}(\tau, 0)$. Therefore $S_{0}$ is precisely the zero set of $w_{\tau}$ and $S_{1}$ the zero set of $z_{\tau}$. Combined with the fact that $w_{\tau} z_{\tau}=p$ and that $p$ is nilpotent, we obtain the results immediately.

Lemma 11. The covering $\{V(\tau)\}$, together with the family of functions $z_{\mathfrak{\tau}}$ and $w_{\mathfrak{\tau}}$, satisfy the conditions of Lemma 4.

Proof. It is a simple matter to check that $\tau_{1} \tau_{2}^{-1} \in S L_{2}\left(\mathbf{Z}_{p}\right)$ if and only if $L_{0}\left(\tau_{1}\right)=L_{0}\left(\tau_{2}\right)$, and $\tau_{1} \tau_{2}^{-1} \in m^{-1} S L_{2}\left(Z_{p}\right) m$ if and only if $L_{1}\left(\tau_{1}\right)=L_{1}\left(\tau_{2}\right)$. It follows immediately that if $\tau_{1} \tau_{2}^{-1} \in \Gamma^{0}(p)$ then $V\left(\tau_{1}\right)=V\left(\tau_{2}\right)$.

Now suppose that $\tau_{1} \tau_{2}^{-1} \notin \Gamma^{0}(p)$ and that $P \in V\left(\tau_{1}\right) \cap V\left(\tau_{2}\right)$. Then

$$
\begin{aligned}
\eta_{P}^{0} & \supseteq \rho\left(L_{0}\left(\tau_{1}\right)+L_{0}\left(\tau_{2}\right)\right) \\
\Pi \eta_{P}^{1} & \supseteq \rho\left(L_{1}\left(\tau_{1}\right)+L_{1}\left(\tau_{2}\right)\right) .
\end{aligned}
$$

Comparing this with lemma 6, we see that we must have either $L_{0}\left(\tau_{1}\right)=L_{0}\left(\tau_{2}\right)$ (if $P \in S_{0}$ ) or $L_{1}\left(\tau_{1}\right)=L_{1}\left(\tau_{2}\right)$ (if $P \in S_{1}$.) In the first case, $\tau_{1} \tau_{2}^{-1} \in S L_{2}\left(\mathbf{Z}_{p}\right)$, while in the second $\tau_{1} \tau_{2}^{-1} \in m S L_{2}\left(\mathbf{Z}_{p}\right) m^{-1}$ where

$$
m=\left(\begin{array}{ll}
0 & p \\
1 & 0
\end{array}\right)
$$

Thus intersections between the $V(\tau)$ can only occur under the proper conditions.

Let us consider the situation when $L_{0}\left(\tau_{1}\right)=L_{0}\left(\tau_{2}\right)$ but $L_{1}\left(\tau_{1}\right) \neq L_{1}\left(\tau_{2}\right)$. Then $L_{1}\left(\tau_{1}\right)+L_{1}\left(\tau_{2}\right)=L_{0}\left(\tau_{1}\right)$ and it follows that

$$
V\left(\tau_{1}\right) \cap V\left(\tau_{2}\right)=\left\{P \in V\left(\tau_{i}\right): \Pi \eta_{P}^{1}=\eta_{P}^{0}\right\} .
$$

Therefore $V\left(\tau_{1}\right) \cap V\left(\tau_{2}\right)=S_{0}\left(\tau_{1}\right)-S_{1}\left(\tau_{1}\right)$ by Lemma 6 and, by Lemma 10 ,

$$
V\left(\tau_{1}\right) \cap V\left(\tau_{2}\right)=V\left(\tau_{i}\right)\left[z_{\tau_{i}}^{-1}\right] .
$$

A similar argument gives the analogous result when $L_{1}\left(\tau_{1}\right)=L_{1}\left(\tau_{2}\right)$.

We now need only show that $\tau_{1} \tau_{2}^{-1} \in S L_{2}\left(\mathbf{Z}_{p}\right)$, then $\tau_{1} \tau_{2}^{-1} z_{\tau_{2}}=z_{t_{1}}$ on $V\left(\tau_{1}\right) \cap V\left(\tau_{2}\right)$, while if $\tau_{1} \tau_{2}^{-1} \in m^{-1} S L_{2}\left(\mathbf{Z}_{p}\right) m$, then $w_{\tau_{1}}=m^{-1} \tau_{1} \tau_{2}^{-1} m w_{\tau_{2}}$ on the intersection. These relations hold as a formal consequence of the definitions of the $z_{\tau}$ and $w_{\mathrm{r}}$.

We have shown that, if $(\eta, T, u, \rho)$ is a set of compatible data on $S$, then there is a morphism $\Phi: S \rightarrow \hat{\mathscr{H}}_{p}$ associated to this data.

\section{Drinfeld's data in the rigid category}

So far, we have shown that Drinfeld's data leads to maps into $\hat{\mathscr{H}}_{p}$. Now we go on to show that a map from $S$ into $\widehat{\mathscr{H}}_{p}$ induces compatible data on $S$ by constructing universal compatible data on $\widehat{\mathscr{H}}_{p}{ }_{p}$. Actually, we return to the rigid space $\mathscr{H}_{p}$ and construct a rigid version of the data $(\eta, T, u, \rho)$ described by Drinfeld. Then we show how to derive the "formal" version on $\hat{\mathscr{H}}_{p}$.

Let $V^{0}$ and $V^{1}$ be constant sheaves of one dimensional $\mathbf{Q}_{p}$ vector spaces on $\mathscr{H}_{p}$, 
with bases $t_{0}$ and $t_{1}$ respectively. Define two invertible sheaves $T^{0}$ and $T^{1}$ by setting

$$
T^{i}=\mathcal{O}_{\mathscr{P}_{p}} \otimes V^{i}
$$

We let $S L_{2}\left(\mathbf{Q}_{p}\right)$ act on $T^{0}$ by the "modular cocyle," so that $\tau^{*} f(z) t_{0}=$ $(c z+d) f(\tau z) t_{0}$. We let $S L_{2}\left(\mathbf{Q}_{p}\right)$ act on $T^{1}$ by the formula $\tau^{*} f(z) t_{1}=$ $(a+b / z) f(\tau z) t_{1}$.

For $i=0,1$, let $\eta^{i}$ be a constant sheaf of two-dimensional $\mathbf{Q}_{p}$-vector spaces on $\mathscr{H}_{p}$ with basis $x_{i 0}$ and $x_{i 1}$. Define a map $u^{0}: \eta^{0}=\mathbf{Q}_{p} x_{00}+\mathbf{Q}_{p} x_{01} \rightarrow T^{0}$ by setting $u^{0}\left(x_{00}\right)=z t_{0}$ and $u^{0}\left(x_{01}\right)=t_{0}$. Define $u^{1}: \eta^{1} \rightarrow T^{1}$ by setting $u^{1}\left(x_{11}\right)=(p / z) t_{1}$ and $u^{1}\left(x_{10}\right)=t_{1}$. Let $S L_{2}\left(\mathbf{Q}_{p}\right)$ act on $\eta$ so that $u$ commutes with the group action. This means that

and

$$
\tau^{*}\left(\begin{array}{c}
x_{00} \\
x_{01}
\end{array}\right)=\left(\begin{array}{ll}
a & b \\
c & d
\end{array}\right)\left(\begin{array}{l}
x_{00} \\
x_{01}
\end{array}\right)
$$

$$
\tau *\left(\begin{array}{c}
x_{10} \\
x_{11}
\end{array}\right)=\left(\begin{array}{cc}
a & b / p \\
p c & d
\end{array}\right)\left(\begin{array}{c}
x_{10} \\
x_{11}
\end{array}\right) .
$$

Let $\Pi$ satisfy the relation $\Pi^{2}=p$. We establish a $Z_{p}[\Pi]$ action on $T=T^{0} \oplus T^{1}$ by declaring that $\Pi t_{0}=(p / z) t_{1}$ and $\Pi t_{1}=z t_{0}$. There is a unique action of $\Pi$ on $\eta=\eta^{0} \oplus \eta^{1}$ such that $u=u^{0} \oplus u^{1}$ commutes with $\Pi$; we let $\mathbf{Z}_{p}[\Pi]$ act on $\eta$ in this way. Then the action of $\Pi$ commutes with the $S L_{2}\left(\mathbf{Q}_{p}\right)$ action as well.

\section{Norms}

In the previous section we constructed $\mathbf{Z} / 2 \mathbf{Z}$-graded sheaves $T=T^{0} \oplus T^{1}$ and $\eta=\eta^{0} \oplus \eta^{1}$ together with a graded map $u=u^{0} \oplus u^{1}$ between them. We would like to describe models for this data on $\hat{\mathscr{H}}_{p}$. In order to do this, we place additional structure on this data.

Definition 6. Let $X$ be a set with a Grothendieck topology, and let $F$ be a sheaf of $\mathbf{Q}_{p}$ vector spaces on $X$. Then a norm on $F$ is a choice, for every open set $U$, of a norm on $F(U)$ which extends the usual norm on $\mathbf{Q}_{p}$. We require that

1. If $V \subset U$, then $|s|_{V} \leqq|s|_{U}$.

2. If $\left\{U_{i}\right\}_{i \in I}$ is an admissible covering of $U$, and $s \in F(U)$, then $|s|_{U} \leqq \max _{i \in I}|s|_{U_{i}}$.

Suppose that $X$ is a rigid analytic space with a formal structure, and that $F$ is a normed sheaf on $X$. The norm enables us to obtain a sheaf $\hat{F}$ on $\hat{X}$ by defining

$$
\hat{F}(U)=\left\{s \in F\left(r^{-1}(U)\right):|s|_{U} \leqq 1\right\}
$$

for open sets $U$ in $\hat{X}$.

Let us now define a norm on the sheaves $T^{0}$ and $T^{1}$. We impose the following conditions.

1. If $U \subset U(\tau)$ is an affinoid, then $\left|\tau^{*} t_{i}\right|_{U}=1$.

2. If $U$ is an affinoid, $f$ is a function on $U$ and $s=f t_{i}$ is a section in $T^{i}(U)$, then $|s|_{U}=|f|\left|t_{i}\right|_{U}$ where $|f|$ is the usual sup-norm for functions. 
3. If $U$ is an affinoid, let $I$ be the set of $\tau \in S L_{2}\left(\mathbf{Q}_{p}\right)$ such that $U(\tau) \cap U \neq \varnothing$. Then

$$
|s|_{U}=\max _{\tau \in I}|s|_{U(\tau) \cap U}
$$

That this gives a well defined norm on $T^{i}$, and that the sheaves $\hat{T}^{i}$ are invertible, follows from the following simple lemma.

Lemma 12. Suppose $V=U\left(\tau_{1}\right) \cap U\left(\tau_{2}\right) \neq \varnothing$. Then the transition function $u=\left(\tau_{1}^{*} t_{i}\right) /\left(\tau_{2}^{*} t_{i}\right)$ satisfies $|u|_{V}=1$.

The sheaf $\eta$ is a subsheaf of $T$. We impose a norm on $\eta$ by setting $|s|_{U}=|u(s)|_{U}$ for any affinoid $U$ and $s \in \eta(U)$.

Definition 7. Let $\hat{T}$ and $\hat{\eta}$ be the sheaves on $\hat{\mathscr{H}}_{p}$ deduced from $T$ and $\eta$ by means of their norms, as in equation (4). Let $\hat{u}$ be the map obtained by restricting $u$ to $\hat{T}$ and $\hat{\eta}$. If

$$
l: \hat{\eta}^{0} \rightarrow \eta^{0}
$$

denotes the inclusion, then

$$
l \otimes 1: \hat{\eta}^{0} \otimes \mathbf{Q}_{p} \rightarrow \eta^{0}
$$

is an isomorphism. Let $\rho=(l \otimes 1)^{-1}$.

Passage from the rigid to the formal category

We will now see that this data is Drinfeld's universal data.

Lemma 13. The data we have constructed earlier on $\widehat{\mathscr{H}}_{p}$ meet Drinfeld's conditions (1), (2), (3), and (4). (See p. 652). More precisely:

1. Let $\hat{\eta}$ and $\hat{T}$ be the sheaves on $\hat{\mathscr{H}}_{p}$ deduced from the norms on $\eta$ and $T$. Then $\hat{\eta}$ and $\hat{T}$ meet requirements (1) and (2).

2. The restriction of $u$ to $\hat{\eta}$ defines a map $\hat{u}: \hat{\eta} \rightarrow \hat{T}$ which meets requirement (3).

3. If $l: \hat{\eta} \rightarrow \eta$ denotes the inclusion map, then $l^{-1}=\rho$ meets requirement (4).

Proof. To show that $\hat{T}$ is a sheaf of $\mathcal{O}_{\hat{\mathscr{H}}_{p}}[\Pi]$ modules, we must verify that, if $U$ is an open set in $\hat{\mathscr{H}}_{p}$ and $s \in T\left(r^{-1}(U)\right)$ satisfies $|s| \leqq 1$, then $|\Pi s| \leqq 1$. On the open set $\bar{U}(\tau)=r(U(\tau)), \quad(c(\tau) z+d(\tau)) t_{0}$ generates $\widehat{T}^{0}(\widetilde{U}(\tau))$. Now $\Pi(c z+d) t_{0}=$ $(c z+d)(p / z) t_{1}$. We compute

$$
\begin{aligned}
\left|(c z+d)(p / z) t_{1}\right|_{U(\tau)} & =\left|(c z+d)(p / z)(z /(a z+b)) \tau^{*} t_{1}\right|_{U(\tau)} \\
& =\left|p(c z+d) /(a z+b) \tau^{*} t_{1}\right|_{U(\tau)} \\
& =|p /(\tau z)|_{U(\tau)} \\
& \leqq 1
\end{aligned}
$$

A similar calculation shows that $\Pi \hat{T}^{1} \subset \hat{T}^{0}$.

On an open set $\bar{U}$ of $\hat{\mathscr{H}}_{p}$, we see that

$$
\hat{\eta}^{0}(\bar{U})=\left\{\alpha x_{00}+\beta x_{01}:\left|(\alpha z+\beta) t_{0}\right|_{U} \leqq 1\right\} .
$$


where $U=r^{-1}(\bar{U})$. Since $\hat{\eta}(\bar{U})$ consists of the elements of a two-dimensional vector space over $\mathbf{Q}_{p}$ of norm less than one with respect to some norm, we see that $\hat{\eta}^{0}(\bar{U})$ is a two-dimensional $\mathbf{Z}_{p}$ lattice. The same argument applies to $\hat{\eta}^{1}$.

By construction, we see that $u(\hat{\eta}) \subset \hat{T}$. On any $U(\tau)$, we see that

$$
u\left(c(\tau) x_{01}+d(\tau) x_{00}\right)=(c z+d) t_{0}=\tau^{*} t_{0},
$$

so that $u \otimes 1: \hat{\eta}^{0} \otimes \mathcal{O} \rightarrow T^{0}$ is surjective. A similar argument gives the result for $\hat{\eta}^{1}$. It is not hard to verify that $\hat{\eta}$ is a $Z_{p}[\Pi]$ module, and, finally, the map $\rho$ identifies $\hat{\eta}^{0} \otimes \mathbf{Q}_{\underline{p}}$ with $\underline{\mathbf{Q}_{p}} \oplus \underline{\mathbf{Q}_{p}}$ by construction.

Our remaining task is to check the compatibility conditions. We begin by indentifying the subsets $S_{0}$ and $S_{1}$ of $\ddot{\mathscr{H}}_{p}$. Notice that these sets are closed subsets of $\hat{\mathscr{H}}_{p}$.

Lemma 14. Let

Then

$$
\begin{aligned}
& S_{0}(\tau)=r(\{P \in U(\tau):|p|<|\tau z(P)| \leqq 1\}) \\
& S_{1}(\tau)=r(\{P \in U(1):|p| \leqq|\tau z(P)|<1\}) .
\end{aligned}
$$

$$
S_{i}=\bigcup_{\tau \in S L_{2}\left(Q_{p}\right)} S_{i}(\tau)
$$

Proof. On the set $\bar{U}(1)=r(U(1))$, we know that $\Pi t_{0}=(p / z) t_{1}$ and that $\Pi t_{1}=z t_{0}$. Therefore the zeros of $\Pi$ on $\widetilde{U}(1)$ are defined by the regions where $p / z$ and $z$ are nonunits in $\mathcal{O} \bar{U}(1)$. These are respectively the reductions of the sets in $\mathscr{H}_{p}$ where $|p / z|<1$ and $|z|<1$. The group action enables us to deduce that Drinfeld's sets $S_{0}$ and $S_{1}$ on the formal scheme $\hat{\mathscr{H}}_{p}$ are

$$
S_{i}=\bigcup_{\tau \in S L_{2}\left(Q_{p}\right)} S_{i}(\tau)
$$

Corollary 15. A point $P$ of $\hat{\mathscr{H}}_{p}$ is rational over $\mathbf{F}_{p}$ if and only if it belongs to $S_{0} \cap S_{1}$.

Proof. From the lemma, we see that $\left(S_{0} \cap S_{1}\right) \cap \bar{U}(1)$ is the reduction of the annulus $\{P:|p|<|z|<1\}$. This is precisely the set of points which reduce to the double point at the rational point of $\bar{U}(1)$. Then the group action, which permutes the rational points of $\hat{\mathscr{H}}_{p}$ transitively, gives the result.

We further extend our understanding of the sets $S_{0}$ and $S_{1}$ in the following lemma.

Lemma 16. Let us say that a vertex $v(\tau)$ of the tree $\mathscr{T}$ is even or odd according to whether ord $(\operatorname{det}(\tau))$ is even or odd. Then

$$
\begin{aligned}
& \hat{\mathscr{H}}_{p}-S_{0}=\bigcup_{v \text { odd }} r\left(r_{\mathscr{F}}^{-1}(v)\right) \\
& \hat{\mathscr{H}}_{p}-S_{1}=\bigcup_{v \text { even }} r\left(r_{\mathscr{S}}^{-1}(v)\right) .
\end{aligned}
$$

Here one should recall that $r_{\mathscr{T}}^{-1}(v)$ is an affinoid and that $r\left(r_{\mathscr{T}}^{-1}(v)\right)$ is a Zariski open set in the reduction of $\mathscr{H}_{p}$ and therefore in $\hat{\mathscr{H}}_{p}$.

Proof. A point $P$ belongs to $\hat{\mathscr{H}}_{p}-S_{0}$ provided that the map $\Pi: \hat{T}^{0} \rightarrow \hat{T}^{1}$ is non- 
zero. On $r(U(1))$, this means that $|p / z(P)|=1$, and therefore $U(1)-S_{0}(1)=$ $\{P \in U(1):|z(P)|=|p|\}$. This set is the inverse image of the vertex corresponding to the linear fractional transformation $z \mapsto p / z$; this vertex is clearly odd. Translation by $S L_{2}\left(Q_{p}\right)$ gives the complete result. The same type of argument works for $\hat{\mathscr{H}}_{p}-S_{1}$.

We are now in a position to establish the compatility conditions.

Lemma 17. The data $\hat{\eta}, \hat{T}, \hat{u}$, and $\rho$ satisfy conditions (C1), (C2), and (C3) on $\hat{\mathscr{H}}_{\mathrm{p}}$.

Proof. Because of the group action, to establish (C1) it suffices to show that $\hat{\eta}^{0} \operatorname{lr}_{\text {(So(1)) }}$ and $\left.\hat{\eta}^{1}\right|_{r\left(S_{1}(1)\right)}$ are constant sheaves isomorphic to $\underline{\mathbf{Z}}_{\underline{p}} \oplus \underline{\mathbf{Z}_{p}}$. This is actually quite simple. If $\bar{U} \subset S_{0}(1)$ is an open set, then

$$
\hat{\eta}^{0}(\bar{U})=\left\{\alpha x_{00}+\beta x_{01}:\left|(\alpha z+\beta) t_{0}\right|_{U}=1\right\} .
$$

where $U$ is the set in $\mathscr{H}_{p}$ which reduces to $\vec{U}$. But $\left|t_{0}\right|_{U}=1$ and, for all points $P \in S_{0}(1)$ we know that $|\alpha z+\beta|=\max (|\alpha||z(P)|,|\beta|)$. (Remember that on this set $z(P)$ is never rational by the corollary above, so 1 and $z(P)$ are linearly independent over $\mathbf{Q}_{p}$.) Since on $S_{0}$ we know that $|z(P)|>|p|$ and $\beta$ is constrained to lie in $\mathbf{Q}_{p}$, we see that $\hat{\eta}^{0}(U)$ is the constant sheaf $\mathbf{Z}_{p} x_{00}+\mathbf{Z}_{p} x_{01}$. A similar argument shows that $\hat{\eta}^{1}=\mathbf{Z}_{p} x_{10}+\mathbf{Z}_{p} x_{11}$.

To show (C2) we must consider three separate cases. A point $P \in \hat{\mathscr{H}}_{p}$ belongs either to $S_{0}-S_{1}$, to $S_{1}-S_{0}$, or to $S_{0} \cap S_{1}$. As usual, the group action allows us to restrict our attention to points in $\bar{U}(1)$. Suppose therefore that $P \in S_{0}(1)-S_{1}(1)$. Then $|z|=1$ on the residue class of $\mathscr{H}_{p}$ defined by $P$. Referring to the previous paragraph, we see that $\hat{\eta}_{P}^{0}=\mathbf{Z}_{p} x_{00}+\mathbf{Z}_{p} x_{01}$. Furthermore,

Therefore

$$
\hat{\eta}_{P}^{1}=\left\{\alpha x_{10}+\beta x_{11}:\left|(\alpha+\beta p / z(P)) t_{1}\right|=1\right\}
$$

$$
\hat{\eta}_{P}^{1}=\mathbf{Z}_{p} x_{10}+\mathbf{Z}_{p}\left(x_{11} / p\right)
$$

We conclude that $\Pi \hat{\eta}_{P}^{1}=\hat{\eta}_{P}^{0}$ and that $\hat{\eta}_{P}^{1} / \Pi \hat{\eta}_{P}^{0}$ is a two dimensional $\mathbf{F}_{p}$ vector space. On the other hand, since $P$ is not a rational point, we know that $\left(T^{1} / \Pi T^{0}\right) \otimes \kappa(P)$ is an extension field of $\mathbf{F}_{p}$. The images under $u$ of $x_{10}$ and $x_{11} / p$ are $t_{1}$ and $t_{1} / z(P)$ respectively, and these are linearly independent over $\mathbf{F}_{p}$. This proves the injectivity of the map in this case. The situation where $P \in S_{1}(1)-S_{0}(1)$ is essentially the same. In the third case, $P$ belongs to $S_{0}(1) \cap S_{1}(1)$, and is a rational point of $\hat{\mathscr{H}}_{p}$. In this case, $\widehat{T}^{i} / \Pi \hat{T}^{i+1}$ is a $\mathbf{l}$-dimensional vector space over $\mathbf{F}_{p}$ for $i=0$ and $i=1$. Using what we know about $\hat{\eta}$, and in particular property $(\mathrm{Cl})$ which we proved earlier, it is not hard to check that each $\hat{\eta}^{i} / \Pi \hat{\eta}^{i+1}$ is 1-dimensional and that again the map is injective.

Finally, we must establish property (C3). On $U(1)$ this property can be checked explicitly since we have computed all of the relevant invariants. Then the general statement follows from the $S L_{2}\left(Q_{p}\right)$ equivariance of our data.

Corollary 18. Let $S$ be a scheme on which $p$ is nilpotent. Let $\Psi: S \rightarrow \hat{\mathscr{H}}_{p}$ be a morphism. Then $\left(\Psi^{-1} \hat{\eta}, \Psi^{*} \hat{T}, \Psi^{-1} u, \Psi^{-1} \rho\right)$ is compatible data on $S$.

Finally, we can obtain the goal of this entire exercise so far: 
Theorem 19. If $D=(\eta, T, u, \rho)$ is compatible data on $S$, let $\Psi_{D}$ be the associated map $S \rightarrow \hat{\mathscr{H}}_{p}$. If $\Psi$ is a map $S \rightarrow \widehat{\mathscr{H}}_{p}$, let $\mathbf{D}(\Psi)$ be the induced data on $S$. Then $\mathbf{D}\left(\Psi_{D}\right)=D$ and $\Psi_{\mathbf{D}(\Psi)}=\Psi$.

Proof. This equivalence can be obtained by laboriously checking all of the various definitions we have made. Since this would amount to repeating much of what has gone before, we leave it to the reader.

\section{More on the sheaf $\hat{T}$}

It follows from Drinfeld's theorem on moduli that $\widehat{T}$ is the tangent space to a family of formal groups on $\hat{\mathscr{H}}_{p}$. To better understand this family, it is worthwhile to describe $\widehat{T}$ in some detail. Our construction of $\hat{T}$ as the integral elements in the normed sheaf $T$ on the rigid space $\mathscr{H}_{p}$ make this relatively simple.

In our construction of $T$, we fixed sections $t_{0}$ and $t_{1}$. Let us define functions $\mu_{i}$ on the tree $\mathscr{T}$ by the formula

$$
\mu_{i}(v)=-\log _{p}\left|t_{i}\right|_{r^{-1}(v)} \cdot
$$

The functions $\mu_{i}$ measure the rate of growth of the sections $t_{i}$ at points approaching the "boundary" of $\mathscr{H}_{p}$. In addition, we may as well consider only $\mu_{0}$, since it is not hard to verify that

$$
\mu_{1}(v(\tau))=\mu_{0}(v(\tau m))
$$

Our choice of a function $z$ on $\mathscr{H}_{p}$ enables us to orient the tree $\mathscr{T}$ so that the "head" of every edge points toward the point at infinity. Call this orientation $w_{\infty}$. Let $d(v)$ be the function on $\mathscr{T}$ which assigns to a vertex $v$ the signed distance from the base vertex $v(1)$ to $v$, computed with respect to this orientation.

In addition to this orientation on $\mathscr{T}$, there are two choices of an $S L_{2}\left(\mathbf{Q}_{p}\right)$ invariant orientation. Let us denote by $w_{0}$ the $S L_{2}\left(\mathbf{Q}_{p}\right)$-invariant orientation which agrees with $w_{\infty}$ on the edge joining $v(1)$ and $v(m)$.

Recall that we can identify the edges of $\mathscr{T}$ with the elements of the coset space $\Gamma^{\mathbf{0}}(p) \backslash S L_{2}\left(\mathbf{Q}_{p}\right)$. We write $e(\tau)$ for the edge corresponding to $\tau$. We select some coset representatives in the next lemma.

Definition 8. If $R$ is a ring, let $U^{0}(p)(R)$ denote the group of invertible matrices in $M_{2}(R)$ having unit determinant and upper right entry divisible by $p$.

Lemma 20. Suppose $X \in M_{2}(R)$ where $R=W\left(\mathbf{F}_{q}\right)$ is an unramified extension of $\mathbf{Z}_{p}$. Then there exists a unique matrix $e \in U^{0}(p)(R)$ such that

Case 1. $\mathrm{eX}$ is of the form

$$
e X=\left(\begin{array}{cc}
\lambda & p^{i} \\
-p^{j} & 0
\end{array}\right)
$$

where $\lambda \neq 0$ and $\lambda=\sum_{k=0}^{j}\left\langle r_{\mathbf{k}}\right\rangle p^{k}$, for elements $r_{k} \in \mathbf{F}_{q}$. 
Case 2. $e X$ is of the form

$$
e X=\left(\begin{array}{cc}
p^{i} & 0 \\
\lambda & p^{j}
\end{array}\right)
$$

where $\lambda=\sum_{k=0}^{j-1}\left\langle r_{k}\right\rangle p^{k}$ and $r_{k} \in \mathbf{F}_{q}$.

We leave to the reader the verification of the following simple fact.

Lemma 21. If $\tau \in S L_{2}\left(\mathbf{Q}_{p}\right)$ is equivalent to a (multiple of a) representative in Case 1 of Lemma 20 , then $w_{0}(e(\tau))=w_{\infty}(e(\tau))$; otherwise $w_{0}(e(\tau))=-w_{\infty}(e(\tau))$.

Let us call an edge $e(\tau)$ with $\tau$ in case 1 even and with $\tau$ in case $2 o d d$. Let $\chi_{0}$ be the function on the edges of $\mathscr{T}$ defined by

$$
\chi_{0}(e)= \begin{cases}1 & w_{0}(e) \neq w_{\infty}(e) \\ 0 & w_{0}(e)=w_{\infty}(e) .\end{cases}
$$

Using this, we determine $\mu_{0}$.

Proposition 22. The function $\mu_{0}$ satisfies

$$
\mu_{0}(v)=\sum_{v(1) \rightarrow v} \chi_{0}(e) w_{\infty}(e)
$$

Proof. Recall that

$$
\left|t_{0}\right|_{U(\tau)}=\left|\frac{1}{c z+d}\right|_{U(\tau)} .
$$

If $e(\tau)$ is even, it follows that $\mu_{0}(v(\tau))=\mu_{0}(v(m \tau))$ since $|c z+d|$ is a constant on $U(\tau)$. If $e(\tau)$ is odd, then, although $|c z+d|$ is not constant, we see that $|a z+b|$ is. Recalling the definition of $U(\tau)$, we see that $\mu_{0}(v(m \tau))=\mu_{0}(v(\tau))+1$. This shows that the "derivative" of $\mu_{0}$ is $\chi_{0}$, as claimed.

To put this result in perspective, let us interpret it in terms of "asymptotics" for the sections $t_{i}$. As is well known, we can identify the ends of $\mathscr{T}$ with the points of $\mathbf{Q}_{p} \cup\{\infty\}$. Suppose that $x \in \mathbf{Q}_{p}$ and $p(x)$ is the corresponding half line in $\mathscr{T}$ originating at $v(1)$.

Corollary 23. If $x \in \mathbf{Q}_{p} \cup\{\infty\}$, then function $\mu_{0}$ satisfies:

$$
\lim _{v \rightarrow x} \frac{\mu_{0}(v)}{d(v)}=\left\{\begin{array}{rll}
-\frac{1}{2} & \text { if } & x \in \mathbf{Q}_{p} \\
\frac{1}{2} & \text { if } & x=\infty
\end{array}\right.
$$

where the limit is taken over the sequence of vertices $v$ in $p(x)$.

Proof. First, notice that the even and odd edges in $\mathscr{T}$ alternate. Then as $x \rightarrow \infty$, every other edge gets counted positively in $\mu_{0}$, since $p(\infty)$ heads "towards infinity." If 
$x \in \mathbf{Q}_{p}$, then eventually $p(x)$ heads "away from infinity." Therefore $\mu_{0}$ counts every other edge negatively.

Corollary 24. The sheaves $\hat{T}$ and $\hat{T}^{-1}$ have no non-zero global sections.

Proof. Suppose $s$ is a section of $\hat{T}^{0}$. Write such a section as $f_{0}(z) t_{0}$ for some rigid function $f_{0}$. We know from rigid geometry that $-\log _{p}|f|$ grows linearly, with integer slope, along half lines $p(x)$. It therefore follows from the previous corollary that $f$ must be bounded as $v \rightarrow \infty$. On the other hand, $f$ must get arbitrarily small along $p(x)$ for $x \in \mathbf{Q}_{p}$, and therefore $f=0$.

If $f_{0} t_{0}^{-1}$ is a section of $\hat{T}^{-1}$, then once again we see that $f$ must be bounded, since otherwise it would grow too fast. But $f$ must vanish at infinity, and therefore $f=0$.

Recall that $\mu_{1}(v(\tau))=\mu_{0}(v(\tau m))$. Similar arguments, with 0 playing the role of $\infty$, will therefore work for $\hat{T}^{1}$.

Let $T_{\mathscr{H}_{p}}$ denote the tangent sheaf to $\mathscr{H}_{p} . T_{\mathscr{H}_{p}}$ carries a norm defined by setting $\left|\frac{d}{d \tau z}\right|_{\overrightarrow{\mathscr{g}}^{-1}(\alpha(\tau))}=1$. The associated sheaf on $\hat{\mathscr{H}}_{p}$ is the tangent sheaf $T_{\hat{\mathscr{H}}_{p}}$.

Proposition 25. The map $t_{0} \wedge t_{1} \rightarrow z \frac{d}{d z}$ induces an $S L_{2}\left(\mathbf{Q}_{p}\right)$-equivariant isomorphism of normed sheaves $\wedge^{2} T \rightarrow T_{\mathscr{*}_{p}}$ and therefore

$$
\stackrel{2}{\wedge} \hat{T}=T_{\hat{\mathscr{H}}_{p}} .
$$

Proof. $T \wedge T$ is spanned by $t_{0} \wedge t_{1}$; it is normed by setting

Observe that

$$
\left|t_{0} \wedge t^{1}\right|_{U}=\left|t_{0}\right|_{U}\left|t_{1}\right|_{U} \text {. }
$$

$$
\tau^{*}\left(t_{0} \wedge t_{1}\right)=\frac{(a z+b)(c z+d)}{z} t_{0} \wedge t_{1} .
$$

The given map therefore commutes with the $S L_{2}\left(\mathbf{Q}_{p}\right)$ action. Next, recall that

$$
d(v)=-\log _{p}\left|\frac{a d-b c}{(c z+d)^{2}}\right|_{r_{\mathscr{g}}^{-1}(v(\tau))} .
$$

It is not hard to deduce from this and the definitions of the group action that

$$
\begin{aligned}
-\log _{p}\left|t_{0} \wedge t_{1}\right|_{r_{\bar{g}}(v(\tau))} & =\mu_{0}(v(\tau))+\mu_{1}(v(\tau)) \\
& =-\log _{p}\left|z \frac{d}{d z}\right|_{r_{\bar{g}} i_{(v(\tau))}} . \square
\end{aligned}
$$

\section{The moduli problem}

\section{A useful SFD-module}

In order to describe Drinfeld's moduli problem, we need to choose a fixed SFD module $\Phi$ over $\overline{\mathbf{F}}_{p}$. We define $\Phi$ by specifying its Dieudonne module. Recall that 
$A \subset \mathcal{O}_{D}$ is a copy of the unramified extension of $\mathbf{Z}_{p}$ of degree 2, and that we have labelled the two embeddings $A \rightarrow W\left(\overline{\mathbf{F}}_{p}\right)$ with $\sigma_{0}$ and $\sigma_{1}$.

Definition 9. Let $M$ be the $W\left(\overline{\mathbf{F}}_{p}\right)[\mathbf{F}, \mathbf{V}]$ module with $\mathbf{V}$-basis $g^{0}$ and $g^{1}$ satisfying the relations $\mathbf{F} g^{i}=\mathbf{V} g^{i}$ OO $_{D}$ acts on $M$ via the rules $\Pi g^{i}=\mathbf{V} g^{i}$ and $l(a) g^{i}=\sigma_{i}(a) g^{i}$ for $a \in A$. Let $\Phi$ be the SFD module such that $\mathbf{M}(\Phi)=M$.

Suppose $m$ is an endomorphism of $\Phi$. Then $m$ induces a linear endomorphism of $\mathbf{M}(\Phi)$ which preserves the grading and commutes with the actions of $\mathbf{F}, \mathbf{V}$, and $\Pi$. We associate a matrix $T(m)$ to $m$ by the rule

$$
\left(\begin{array}{c}
m\left(g^{0}\right) \\
m\left(\mathbf{V} g^{1}\right)
\end{array}\right)=T(m)^{-1}\left(\begin{array}{c}
g^{0} \\
\mathbf{V} g^{1}
\end{array}\right)
$$

Lemma 26. The map $T: \operatorname{End}(\Phi) \rightarrow \mathbf{M}_{2}\left(W\left(\overline{\mathbf{F}}_{p}\right)\right)$ establishes an isomorphism between End $(\Phi)$ and the subring of $M_{2}\left(Z_{p}\right)$ consisting of matrices with lower left entry divisible by $p$.

Proof. Since $m \in E$ nd $(\Phi)$ must commute with $\mathbf{F}$ and $\mathbf{V}$, we must have $T(m) \in M_{2}\left(\mathbf{Z}_{p}\right)$. The condition on the lower left entry comes from the fact that $m\left(\mathbf{V} g^{1}\right) \in \mathbf{V M}(\Phi)$. These considerations show that $T(m)$ lies in the desired set. Conversely, if $T$ is a matrix satisfying the stated condition, it uniquely determines an element $m$ of $\operatorname{End}(\Phi)$.

We use this lemma to identify $\operatorname{End}(\Phi) \otimes \mathbf{Q}_{p}$ with $M_{2}\left(\mathbf{Q}_{p}\right)$. We point out that, if $m \in \operatorname{End}(\Phi) \otimes \mathbf{Q}_{p}$, then the height of $m$ is zero if and only if $\operatorname{ord}_{p}(\operatorname{det}(T(m)))=0$.

Remark 27. The SFD-module $\Phi$ can be obtained more directly. Let $G$ be the reduction $\bmod p$ of a Lubin group of height 2 over $W\left(\overline{\mathbf{F}}_{p}\right)$. It is well known that $\operatorname{End}(G)=\mathcal{O}_{D}$. Let $\Phi=G \times G$. We identify $\operatorname{End}(\Phi)$ with $M_{2}(\operatorname{End}(G))$. If $a \in W\left(\overline{\mathbf{F}}_{p}\right)$ is of degree two over $\mathbf{Z}_{p}$, we let $[a]_{G}$ denote the corresponding endomorphism of $G$, so that $[a]_{G}$ acts on the tangent space of $G$ as $a \bmod p$. Then for $a \in A \subset D$, we define

$$
[a]_{\Phi}=\left(\begin{array}{cc}
{\left[\sigma_{0}(a)\right]_{G}} & 0 \\
0 & {\left[\sigma_{1}(a)\right]_{G}}
\end{array}\right) .
$$

Finally, we choose $\Pi \in \operatorname{End}(G)$ so that $\Pi^{2}=p$ and $\Pi[a]_{G}=[\bar{a}]_{G} \Pi$ and we let

$$
[\Pi]_{\Phi}=\left(\begin{array}{cc}
\Pi & 0 \\
0 & \Pi
\end{array}\right)
$$

\section{Rigidified SFD-modules}

We will now recall Drinfeld's moduli problem. (See [Dr, p. 109].) Let Nilp be the category of $\mathbf{Z}_{p}$ algebras on which $p$ is nilpotent. For any $B$ in Nilp, let $\operatorname{SFD}(B)$ denote the set of isomorphism classes of triples $(\psi, G, \rho)$ where:

1. $\psi$ is a homomorphism $\overline{\mathbf{F}}_{p} \rightarrow B / p B$.

2. $G$ is an SFD-module over $B$ of height 4 at each point of $\operatorname{Spec} B / p B$.

3. $\rho: \psi_{*} \Phi \rightarrow G$ is a quasi-isogeny of height zero. 
$G L_{2}\left(\mathbf{Q}_{p}\right) \otimes D^{*}$ acts on SFD. If $\left(g_{1}, g_{2}\right)$ belongs to this group, then

$$
\left(g_{1}, g_{2}\right) \cdot(\psi, G, \rho)=\left(\psi \circ \mathrm{Fr}^{-n}, G^{g_{2}}, \rho g_{1}^{-1} g_{2}^{-1} \mathrm{Frob}^{n}\right)
$$

Here $n\left(g_{1}, g_{2}\right)=\operatorname{ord}_{p}\left(\operatorname{det}\left(g_{1}\right)\right)+\operatorname{ord}_{p}\left(N\left(g_{2}\right)\right), \operatorname{Fr}: \overline{\mathbf{F}}_{p} \rightarrow \overline{\mathbf{F}}_{p}$ is the Frobenius map, and Frob is the natural quasi-isogeny from $\mathrm{Fr}_{*}^{-n} \Phi \rightarrow \Phi$. $G^{g_{2}}$ is the SFD-module $G$ with the endomorphism action conjugated by $g_{2}$.

Now we state Drinfeld's theorem.

Theorem 28. (Drinfeld [Dr, p. 109]) The functor SFD is represented by the formal scheme $\hat{\mathscr{H}}_{p} \otimes S p f W\left(\overline{\mathbf{F}}_{p}\right)$. The action of $G L_{2}(K) \times D^{*}$ on $S F D$ corresponds to the natural action of $P G L_{2}(K)$ on $\hat{\mathscr{H}}_{p}$ and to the action $\left(g_{1}, g_{2}\right) \mapsto F r^{-n\left(g_{1}, g_{2}\right)}$ on $\operatorname{Spf} W\left(\overline{\mathbf{F}}_{p}\right)$.

In practical terms, Drinfeld constructs, for every triple $(\psi, G, \rho)$ over $B$, data $(\eta, T, u, \rho)$ on $\operatorname{Spec} B$ giving a point of $\hat{\mathscr{H}}_{p} \otimes \operatorname{Spf} W\left(\overline{\mathbf{F}}_{p}\right)$. Let us briefly outline his construction, which is described in [Dr, p. 110]. Let $\mathbf{M}=\mathbf{M}(G)$ be the Dieudonne module for $G$, with its grading and $\Pi$ action. Let $\mathbf{N}=\mathbf{M} \times \mathbf{M} / \sim$ where $(\mathbf{V} x, 0) \sim(0, \Pi x)$. Let $\lambda: \mathbf{N} \rightarrow \mathbf{M}$ be the map $\lambda((x, y))=\Pi x+\mathbf{V} y . \mathbf{N}$ carries a grading, and there is a well defined map $\mathbf{N} \rightarrow \mathbf{M} / \mathbf{V M}$ induced by projection onto the first component. Further, there is a canonical, universally defined map $L: \mathbf{M} \rightarrow \mathbf{N}$ such that $\lambda L=\mathbf{F}$. Define $\varphi: \mathbf{N} \rightarrow \mathbf{N}$ by setting $\varphi(x, y)=L(x)+(y, 0)$. Then

1. $\eta=\{x \in \mathbf{N}: \varphi(x)=x\}$.

2. $T$ is the tangent space to $G$; it is canonically isomorphic to $\mathbf{M} / \mathbf{V M}$.

3. $u$ is induced by the projection map $\mathbf{N} \rightarrow \mathbf{M} / \mathbf{V M}$.

4. Fix an isomorphism $\eta^{\mathbf{0}}(\Phi)$ with $\mathbf{Z}_{p} \oplus \mathbf{Z}_{p}$. Then the quasi-isogeny $\rho$ induces

$$
\rho: \mathbf{Q}_{p} \oplus \mathbf{Q}_{p}=\eta^{\circ}\left(\psi_{*} \Phi\right) \otimes \mathbf{Q}_{p} \rightarrow \eta^{\circ}(G) \otimes \mathbf{Q}_{p} .
$$

As we see from this, an essential element in Drinfeld's construction is knowledge of the data $(\eta, T, u, \rho)$ for the basic module $\Phi$. We compute this data now.

\section{Drinfeld's data for $\Phi$}

As a first step in classifying SFD modules over $\overline{\mathbf{F}}_{p}$, let us compute the invariants associated to our fundamental module $\Phi$. This is essential since, as we remarked above, $\eta^{\mathbf{0}}(\Phi) \otimes \mathbf{Q}_{p}$ serves as our constant sheaf $\mathbf{Q}_{p} \oplus \mathbf{Q}_{p}$ when giving the data $(\eta, T, u, \rho)$ for an arbitrary SFD module $G$.

Lemma 29. The map $L: \mathbf{M}(\Phi) \rightarrow \mathbf{N}(\Phi)$ is defined by the relations $L\left(g^{i}\right)=\left[g^{i}, 0\right]$.

Proof. We must compute universally. Suppose that

$$
\begin{aligned}
& \Pi \gamma^{0}=\left\langle x_{0}\right\rangle \gamma^{1}+\mathrm{V}\left\langle x_{1}\right\rangle \gamma^{0}+\cdots \\
& \Pi \gamma^{1}=\left\langle y_{0}\right\rangle \gamma^{0}+\mathrm{V}\left\langle x_{0}\right\rangle \gamma^{1}+\cdots
\end{aligned}
$$

defines an SFD module $\Gamma$ over some $W\left(\overline{\mathbf{F}}_{p}\right)$-algebra $R$ (see [Dr, Proposition 2.1]). We know that $x_{0} y_{0}=p$. We compute

$$
p \gamma^{0}=\langle p\rangle \gamma^{0}+\mathrm{V}\left\langle x_{0}^{p} y_{1}+x_{1} x_{0}\right\rangle \gamma^{1}+\cdots
$$


Therefore

$$
\varepsilon \mathbf{F} \gamma^{0}=\left\langle x_{0}^{p} y_{1}+x_{1} x_{0}\right\rangle \gamma^{1}+\cdots
$$

where $\varepsilon$ is a unit in $W\left(\overline{\mathbf{F}}_{p}\right)$, and indeed $\varepsilon \equiv 1(\bmod p)$. We conclude that

$$
\varepsilon \mathbf{F} \gamma^{0}=\left\langle x_{0}^{p-1} y_{1}+x_{1}\right\rangle \Pi \gamma^{0}+\mathbf{V} u
$$

for some $u \in \mathbf{M}(\Gamma)$. In our situation, we must specialize to $x_{0}=y_{0}=0$ and $x_{1}=y_{1}=1$, with all other $x_{i}$ and $y_{i}$ being zero. We conclude that $L\left(g^{0}\right)=\left[g^{0}, 0\right]$, and $L\left(g^{1}\right)=\left[g^{1}, 0\right]$.

Corollary 30. $\eta^{i}$ for $\Phi$ is spanned over $\mathbf{Z}_{p}$ by $\left[g^{i}, 0\right]$ and $\left[\mathbf{V} g^{i+1}, 0\right]$.

Proof. Using the previous result, we see that $L\left(\mathbf{V} g^{i+1}\right)=\left[\mathbf{V} g^{i+1}, 0\right]$. Then

$$
\varphi\left(\left[x g^{i}+y \mathbf{V} g^{i+1}, z g^{i}+w \mathbf{V} g^{i+1}\right]\right)=\left[\left(x^{\sigma}+z\right) g^{i}+\left(y^{\sigma}+w\right) \mathbf{V} g^{i+1}, 0\right] .
$$

Our element belongs to $\eta$ when $x=x^{\sigma}, y=y^{\sigma}$, and $z=w=0$.

The period function

We can now define a "period function" on SFD, combining our work in Sect. 4 and Drinfeld's construction.

Definition 10. Suppose that $(\psi, G, \rho)$ is a point of $S F D(R)$ where $R$ is any ring in proNilp of characteristic zero. Then we set

$$
z_{i}(\psi, G, \rho)=u\left(\rho\left(\left[g^{i}, 0\right]\right)\right) / u\left(\rho\left(\left[\mathbf{V} g^{i+1}, 0\right]\right)\right) .
$$

Lemma 31. The functions $z_{i}$ defined in (5) are well defined on $S F D(R)$ for any $R$ in proNilp of characteristic zero, take values in the fraction field of $R$, and satisfy $p z_{0} z_{1}=1$. Further, the map

$$
(\psi, G, \rho) \mapsto\left(z_{i}(\psi, G, \rho), \psi\right)
$$

establishes an isomorphism between $\operatorname{SFD}\left(W\left(\overline{\mathbf{F}}_{p}\right)\right)$ and

$$
\left(\hat{\mathscr{H}}_{p} \otimes \operatorname{Spf} W\left(\overline{\mathbf{F}}_{p}\right)\right)\left(W\left(\overline{\mathbf{F}}_{p}\right)\right)=\hat{\mathbf{Q}}_{p}^{u r}-\mathbf{Q}_{p} \times \operatorname{Hom}\left(W\left(\overline{\mathbf{F}}_{p}\right), W\left(\overline{\mathbf{F}}_{p}\right)\right) .
$$

Here we implicitly identify $\operatorname{Hom}\left(\overline{\mathbf{F}}_{p}, \overline{\mathbf{F}}_{p}\right)$ and $\operatorname{Hom}\left(W\left(\overline{\mathbf{F}}_{p}\right), W\left(\overline{\mathbf{F}}_{p}\right)\right)$.

Proof. This follows from our discussion in Sect. 4. We fix the basis $x_{0}=\left[g^{0}, 0\right]$ and $x_{1}=\left[\mathbf{V} g^{1}, 0\right]$ for $\eta^{0}(\Phi)$, thereby identifying $\eta^{0}(\Phi) \otimes \mathbf{Q}_{p}$ with $\mathbf{Q}_{p} \oplus \mathbf{Q}_{p}$. Then comparing with Lemma 5 , we see that $z_{0}$ is just the function defined there as $z_{\tau}$ when $\tau$ is the identity in $S L_{2}\left(Q_{p}\right)$. Some linear fractional transformation of this function is defined for every point in $\operatorname{SFD}(R)$; since we assume $R$ of characteristic zero, $z_{0}$ is defined everywhere. It follows from the action of $\Pi$ that $p z_{0} z_{1}=1$, and therefore $z_{1}$ is defined everywhere as well. Comparing then with the universal data on $\hat{\mathscr{H}}_{p}$, we see that $z_{0}$ can be viewed as a rigid analytic parameter on SFD, establishing an isomorphism between $\operatorname{SFD}\left(W\left(\overline{\mathbf{F}}_{p}\right)\right)$ and $\hat{\mathbf{Q}}_{p}^{u r}-\mathbf{Q}_{p} \times \operatorname{Hom}\left(W\left(\overline{\mathbf{F}}_{p}\right), W\left(\overline{\mathbf{F}}_{p}\right)\right)$ as claimed. 
We emphasize that $z_{0}$ takes values in the fraction field of $R$, and therefore should properly be viewed as a rigid analytic function on $\mathscr{H}_{p}$, rather than as a function on $\hat{\mathscr{H}}_{\boldsymbol{p}}$.

\section{Construction of an SFD module with given period}

Given an $x \in \hat{\mathbf{Q}}_{p}^{u r}-\mathbf{Q}_{p}$ and a $\psi: W\left(\overline{\mathbf{F}}_{p}\right) \rightarrow W\left(\overline{\mathbf{F}}_{p}\right)$, we will now construct a triple $(\psi, G, \rho)$ such that $z_{0}(\psi, G, \rho)=x$. We proceed by first constructing the special fiber of $G$ over $\overline{\mathbf{F}}_{p}$, and then lifting it to $W\left(\overline{\mathbf{F}}_{p}\right)$.

\section{Some "modular forms" on SFD}

The data $(\eta, T, u, \rho)$ associated to a $W\left(\overline{\mathbf{F}}_{p}\right)$-valued point $P$ of $\hat{\mathscr{H}}_{p}$ can be combined

$$
\mathbf{Q}_{p} x_{0}+\mathbf{Q}_{p} x_{1} \stackrel{\rho}{\rightarrow} \eta^{0} \otimes \mathbf{Q}_{p} \stackrel{u}{\rightarrow} T^{0} \otimes \mathbf{Q}_{p},
$$

to yield an injective map

$$
q(P): \mathbf{Q}_{p} x_{0}+\mathbf{Q}_{p} x_{1} \rightarrow \hat{\mathbf{Q}}_{p}^{u r} t_{\mathbf{o}}
$$

where $t_{0}$ denotes a basis for $T^{0}$. In fact, $q(P)$ is an invariant of $P$ up to projective transformations. This is clear, since $q(P)$ and $q(Q)$ are isomorphic precisely when $z(P)=\tau z(Q)$ for $z \in G L_{2}\left(\mathbf{Q}_{p}\right)$, where $z$ is one of the coordinate functions defined in Lemma 5 .

Consider now the quotient space $\hat{\mathscr{H}}_{p}\left(W\left(\overline{\mathbf{F}}_{p}\right)\right) / G L_{2}\left(\mathbf{Q}_{p}\right)$. Elements of this set are precisely the injective maps $\mathbf{Q}_{p} x_{0}+\mathbf{Q}_{p} x_{\mathbf{1}} \rightarrow \hat{\mathbf{Q}}_{p}^{u r}$ up to isomorphism; this in turn is just the set of 2-dimensional $\mathbf{Q}_{p}$ vector spaces $V$ in $\hat{\mathbf{Q}}_{p}^{u r}$, up to the equivalence $V \sim x V$ for $x \in \hat{\mathbf{Q}}_{p}^{u r}$. Let us now classify such subspaces $V$.

Any 2-dimensional $V \subset \hat{\mathbf{Q}}_{p}^{u r}$ will be spanned by two elements $x$ and $y$. Since $x$ and $y$ are independent over $\mathbf{Q}_{p}$, the vectors $[x, y]$ and $\left[x^{\sigma}, y^{\sigma}\right]$ are independent. Therefore we may write

$$
[x, y]=\alpha\left[x^{\sigma}, y^{\sigma}\right]+\beta\left[x^{\sigma^{2}}, y^{\sigma^{2}}\right] .
$$

The following lemma is quite simple to prove:

Lemma 32. The invariants $\alpha$ and $\beta$ do not depend on the choice of basis $x$ and $y$, and are therefore invariants of the space $V \subset \hat{\mathbf{Q}}_{p}^{u r}$. Conversely, given $\alpha$ and $\beta$ in $\hat{\mathbf{Q}}_{p}^{u r}$, the set $[x, y]$ which solve (6) form a two-dimensional $\mathbf{Q}_{p}$ vector space in $\hat{\mathbf{Q}}_{p}^{\text {ur }}$. Finally, if $V$ and $V^{\prime}$ are two 2-dimensional $\mathbf{Q}_{p}$-subspaces of $\hat{\mathbf{Q}}_{p}^{u r}$, then $V=x V^{\prime}$ if and only if $[\alpha(V), \beta(V)]=\left[\alpha(V)^{\sigma-1}, \beta(V)^{\sigma^{2}-1}\right]$.

Corollary 33. Define $i(V)=\alpha(V)^{\sigma+1} / \beta(V)$. Then $V \sim V^{\prime}$ if and only if $i(V)=i\left(V^{\prime}\right)$.

We remark that, as is well known, the quotient of $\mathbf{P}_{\mathbf{F}_{p}}^{1}$ by $P S L_{2}\left(\mathbf{F}_{p}\right)$ is a projective line with a canonical choice of parameter. In a sense, the function $i$ reduces $\bmod p$ to this canonical parameter. To clarify this, we leave to the reader the task of relating $i$ to the cross ratio. 
Suppose now that we consider the subspace $V$ spanned by $1 /\left(x-x^{\sigma}\right)$ and $x /\left(x-x^{\sigma}\right)$, for reasons which will be clearer later. Then we obtain invariants $\alpha$ and $\beta$ which serve as "modular forms."

Definition 11. For elements $x \in \hat{\mathbf{Q}}_{p}^{u r}$, define

$$
\begin{aligned}
& \alpha(x)=\frac{x-x^{\sigma^{2}}}{x-x^{\sigma}} \\
& \beta(x)=-\left(x-x^{\sigma}\right)^{\sigma^{2}-\sigma} .
\end{aligned}
$$

The functions $\alpha(x)$ and $\beta(x)$ defined here are the invariants associated to the $\mathbf{Q}_{p^{-}}$ subspace of $\hat{\mathbf{Q}}_{p}^{u r}$ spanned by $1 /\left(x-x^{\sigma}\right)$ and $x /\left(x-x^{\sigma}\right)$. Also define

$$
j(x)=\operatorname{ord}_{p}\left(x-x^{\sigma}\right) \text {. }
$$

These functions are "modular" with respect to $G L_{2}\left(\mathbf{Z}_{p}\right)$.

Lemma 34. Suppose $\tau \in G L_{2}\left(\mathbf{Z}_{p}\right)$ and

Then

$$
\tau=\left(\begin{array}{ll}
a & b \\
c & d
\end{array}\right)
$$

$$
\begin{aligned}
& \alpha(\tau x)=(c x+d)^{1-\sigma^{2}} \alpha(x) \\
& \beta(\tau x)=(c x+d)^{\sigma-\sigma^{3}} \beta(x) \\
& j(\tau x)=-2 o r d_{p}(c x+d)+j(x) .
\end{aligned}
$$

The significance of these modular forms will become abundantly clear later, but for now let us point out that projectively equivalent points in $\hat{\mathscr{H}}_{p}\left(W\left(\overline{\mathbf{F}}_{p}\right)\right)$ correspond to triples $(\psi, G, \rho)$ in $\operatorname{SFD}\left(W\left(\tilde{\mathbf{F}}_{p}\right)\right)$ which have isomorphic $G$ parts (although possibly different $\rho$ and $\psi$ parts.) As a result, the modular forms $\alpha$ and $\beta$, and the invariant $i$, determine the isomorphism class of $G$ in a triple $(\psi, G, \rho)$ as an SFD-module, disregarding the rigidifying data.

\section{The desired SFD-module $\bmod p$}

We will construct the special fiber of our desired SFD module using Dieudonne modules. The Dieudonne module $\mathbf{M}(G)$ of the $G$ we seek will be a free, rank 4, $W\left(\overline{\mathbf{F}}_{p}\right)$-module, with an action by $\mathbf{F}$ and $\mathbf{V}$ such that $\mathbf{M} / \mathbf{V M}$ is 2-dimensional over $\overline{\mathbf{F}}_{p}$. In addition, $\mathbf{M}(G)$ will carry a grading from the endomorphism action, and an action of $\Pi$. To give the quasi-isogeny $\rho$, it suffices to give a $W\left(\overline{\mathbf{F}}_{p}\right)$-linear map from $\mathbf{M}\left(\psi_{*} \Phi\right)$ to $\mathbf{M}(G)$ which preserves the grading and commutes with the various operators $\Pi, \mathbf{F}$, and $\mathbf{V}$. We point out that the module $\mathbf{M}\left(\psi_{*} \Phi\right)$ is isomorphic to the module obtained from $\mathbf{M}(\Phi)$ by the extension of scalars induced by $\psi$. In concrete terms (see Definition 9), this means that $\mathbf{M}\left(\psi_{*} \Phi\right)$ is the free $W\left(\overline{\mathbf{F}}_{p}\right)$-module on $\left\{g^{0}, \mathbf{V} g^{1}, g^{1}, \mathbf{V} g^{0}\right\}$ with $W\left(\overline{\mathbf{F}}_{p}\right)$ action $a \cdot g^{i}=\psi\left(\sigma_{i}(a)\right) g^{i}$.

We now re-cast our search for $G$ in terms of matrices. Suppose we are given an SFD-module $G$, and that $\gamma=\left\{\gamma^{0}, \gamma^{1}\right\}$ is a $\mathbf{V}$-basis for $\mathbf{M}(G)$. Then we associate to an 
isogeny $\rho: \psi_{*} \Phi \rightarrow G$ matrices $T^{i}(\rho, \gamma)$ by setting

$$
\left(\begin{array}{c}
r\left(g^{i}\right) \\
r\left(\mathbf{V} g^{i+1}\right)
\end{array}\right)=T^{i}(\rho, \gamma)^{t}\left(\begin{array}{c}
\gamma^{i} \\
\mathbf{V} \gamma^{i+1}
\end{array}\right) .
$$

We point out that either one of $T^{0}$ and $T^{1}$ determines the other. We can put the matrices $T^{i}$ into simple forms by changing our $\mathrm{V}$-basis, as we now see.

Lemma 35. Elements $\delta^{0}$ and $\delta^{1}$ of $\mathbf{M}(G) \otimes \mathbf{Q}_{p}$ form a $\mathbf{V}$-basis for $\mathbf{M}(G)$ if and only if

$$
\left(\begin{array}{c}
\delta^{i} \\
\mathbf{V} \delta^{i+1}
\end{array}\right)=X^{i}\left(\begin{array}{c}
\gamma^{i} \\
\mathbf{V} \gamma^{i+1}
\end{array}\right)
$$

for some $X \in U^{0}(p)\left(W\left(\overline{\mathbf{F}}_{p}\right)\right)$.

Proof. We can write, for any $\mathrm{V}$-basis $\delta^{0}$ and $\delta^{1}$, the equations

$$
\begin{aligned}
\delta^{i} & =a \gamma^{i}+b \mathbf{V} \gamma^{i+1} \\
\mathbf{V} \delta^{i+1} & =c \gamma^{i}+d \mathbf{V} \gamma^{i+1} .
\end{aligned}
$$

The matrix $X$ defined by these must have $W\left(\overline{\mathbf{F}}_{p}\right)$-coefficients and be $W\left(\overline{\mathbf{F}}_{p}\right)$ invertible. In order that $\delta^{i+1}$ to belong to $\mathbf{M}(G)$, it is necessary and sufficient that $p$ divide $c$.

Lemma 36. Let $\rho: \psi_{*} \Phi \rightarrow G$ be an isogeny where $G$ is not isomorphic to $\psi_{*} \Phi$. Then there exists a $\mathbf{V}$-basis $\gamma$ for $\mathbf{M}(G)$ so that one of the $T^{i}(\rho, \gamma)$ has the form of lemma 20, Case 1. In the notation of that lemma, we must have $p^{-j}\left(\lambda^{\sigma}-\lambda\right) \in W\left(\overline{\mathbf{F}}_{p}\right)^{\times}$ and therefore $r_{k} \in \mathbf{F}_{p}$ for $k=0, \ldots, j-1$ and $r_{j} \in \overline{\mathbf{F}}_{p}-\mathbf{F}_{p}$.

Proof. Fix some $\mathbf{V}$-basis $\gamma^{\prime}$ for $\mathbf{M}(G)$. Then the previous lemma shows that we may modify $\gamma^{\prime}$ and thereby replace $T^{i}\left(\rho, \gamma^{\prime}\right)$ by $X T^{i}\left(\rho, \gamma^{\prime}\right)$ for any $X \in U^{0}(p)\left(W\left(\overline{\mathbf{F}}_{p}\right)\right)$. Therefore we may find $\gamma$ so that $T^{0}(\rho, \gamma)$ is in one of the special forms of lemma 20. But a simple check shows that if $T^{0}$ has the form of case 2 of that lemma, then $T^{1}$ has the form of case 1 . Now a calculation shows that, if $T^{i}$ is as in case 1 , we have

$$
\begin{aligned}
\Pi \gamma^{i} & =\mathrm{V} \gamma^{i} \\
\Pi \gamma^{i+1} & =p^{-j}\left(\lambda-\lambda^{\sigma}\right) \gamma^{i}+\mathbf{V} \gamma^{i+1}
\end{aligned}
$$

As a result, we must have $p^{-j}\left(\lambda-\lambda^{\sigma}\right) \in W\left(\overline{\mathbf{F}}_{p}\right)^{\times}$.

Lemma 37. Suppose given a map $\psi: W\left(\overline{\mathbf{F}}_{p}\right) \rightarrow W\left(\overline{\mathbf{F}}_{p}\right)$, an element $i \in \mathbf{Z} / 2$, and a matrix

$$
T=\left(\begin{array}{cc}
\lambda & p^{i} \\
-p^{j} & 0
\end{array}\right)
$$

with $\lambda \in W\left(\overline{\mathbf{F}}_{p}\right)$ such that $p^{-j}\left(\lambda-\lambda^{\sigma}\right) \in W\left(\overline{\mathbf{F}}_{p}\right)$ and $i+j$ is even. Then there exists $a$ 
unique set consisting of an SFD-module $G$ over $\tilde{\mathbf{F}}_{p}$, a quasi-isogeny of height zero $\rho: \psi_{*} \Phi \rightarrow G$, and a $\mathbf{V}$-basis $\gamma$ for $\mathbf{M}(G)$, such that $T^{i}(\rho, \gamma)=p^{-(i+j) / 2} T$.

Proof. Let $\gamma^{i}$ and $\gamma^{i+1}$ be the elements of $M\left(\psi_{*} \Phi\right) \otimes \mathbf{Q}_{p}$ which satisfy

$$
\left(\begin{array}{c}
\gamma^{i} \\
\mathbf{V} \gamma^{i+1}
\end{array}\right)=p^{(i+j) / 2}\left(T^{-1}\right)^{t}\left(\begin{array}{c}
g^{i} \\
\mathbf{V} g^{i+1}
\end{array}\right)
$$

Then the $\gamma^{i}$ are a $\mathbf{V}$-basis for the desired SFD-module.

Corollary 38. Let $\psi$ and $i$ be as in the preceeding lemma, and let

$$
T=\left(\begin{array}{ll}
a & b \\
c & d
\end{array}\right) \in S L_{2}\left(\mathbf{Q}_{p}\right) .
$$

If $d / b \in W\left(\overline{\mathbf{F}}_{p}\right)$ and $a^{\sigma} b-b^{\sigma} a \in W\left(\overline{\mathbf{F}}_{p}\right)$ then there is a unique set consisting of $G, r$, and $\gamma$ such that $T^{i}(\rho, \gamma)=T$.

Proof. The conditions on $T$ imply that there is an $e \in U^{0}(p)\left(W\left(\overline{\mathbf{F}}_{p}\right)\right)$ and an $n \in \mathbf{Z}$ so that $p^{n} e T$ has the form described in Lemma 37.

Now we can define the special fiber of the triple we seek.

Definition 12. Let $x \in \hat{\mathbf{Q}}_{p}^{u r}-\mathbf{Q}_{p}$. Let $M(x)$ be the matrix

$$
M(x)=\left(x-x^{\sigma}\right)^{-1}\left(\begin{array}{cc}
x & 1 \\
-x^{\sigma} & -1
\end{array}\right)\left(\begin{array}{ll}
0 & p \\
1 & 0
\end{array}\right)^{j(x)} .
$$

If $\psi: \overline{\mathbf{F}}_{p} \rightarrow \overline{\mathbf{F}}_{p}$ is a homomorphism, let $P(x)$ be the triple $(\psi, G(x), \rho(x))$ where $G(x)$ and $\rho(x)$ are the SFD-module and quasi-isogeny determined by the map $\psi$, the matrix $M(x)$, and the class of $j(x) \bmod 2$ by Corollary 38. Notice that since $\operatorname{ord}_{p}(\operatorname{det}(M(x)))=0$, $P(x)$ is a well-defined point of $\operatorname{SFD}\left(\overline{\mathbf{F}}_{p}\right)$.

Let us now make explicit the structure of $\mathbf{M}(G(x))$. The matrix $M(x)$ gives us a Vbasis $\gamma$ for $\mathbf{M}(G(x))$ via the formula

$$
\left(\begin{array}{c}
g^{j(x)} \\
\mathbf{V} g^{j(x)+1}
\end{array}\right)=M(x)^{t}\left(\begin{array}{c}
\gamma^{j(x)} \\
\mathbf{V} \gamma^{j(x)+1}
\end{array}\right)
$$

Given that this map commutes with $\mathbf{F}, \mathbf{V}$, and $\Pi$, it is not hard to compute the following rules.

Lemma 39. The $\mathbf{V}$-basis defined in (12) satisfies the following relations:

$$
\begin{aligned}
\Pi \gamma^{j(x)} & =p \gamma^{j(x)+1} \\
\mathbf{F} \gamma^{j(x)+1} & =\alpha(x) \gamma^{j(x)}+\mathrm{V} \beta(x) \gamma^{j(x)+1} .
\end{aligned}
$$

Notice that these relations suffice to determine the module $\mathbf{M}(G)$. 
The invariants of $P(x)$

Let us compute the data $(\eta, T, u, \rho)$ for the point $P(x)$ defined above. The following lemma makes our calculation easier.

Lemma 40. Let $G$ be any SFD-module of dimension 2 and suppose $(x, y) \in \mathbf{N}(G)$ satisfies $\varphi(x, y)=(x, y)$. Then $u=\Pi x+\mathbf{V} y$ satisfies $\mathbf{F} u=\Pi u$.

Proof. Write $L(x)=(a, b)$, where $L$ is Drinfeld's map discussed on page 25 . Then, from the definition of $\varphi$, we must have

$$
\Pi(a+y)+\mathbf{v} b=\Pi x+\mathrm{V} y .
$$

Re-arranging gives

$$
\Pi a+\mathrm{V} b+\Pi y=\Pi x+\mathrm{V} y
$$

and therefore

$$
\mathbf{F} x+\Pi y=\Pi x+\mathbf{V} y .
$$

Apply $\Pi$ to both sides of this equation to obtain the result.

It is also worth pointing out the following.

Lemma 41. Suppose $G$ is an $S F D$-module of dimension 2 and that $x \in \Pi \eta(G)$. Then there is a unique $u \in \mathbf{M}(G)$ such that $x=(u, 0)$ in $\mathbf{N}(G)$.

Proof. We can write $x=(a, b)$ in $\mathbf{N}(G)$, with $b \in \Pi \mathbf{M}(G)$. Therefore $b=\Pi v$ for $v \in \mathbf{M}(G)$, and we see that

$$
(a, b)=(a, \Pi v)=(a+V v, 0) .
$$

The uniqueness follows from the injectivity of $\mathrm{V}$ and $\Pi$.

Now let us consider our special situation. Notice first of all that

$$
\Pi \mathbf{M}(G(x))^{j(x)+1}=\mathbf{M}(G(x))^{j(x)} .
$$

Therefore $\Pi \eta^{j(x)+1}=\eta^{j(x)}$ and $\Pi T^{j(x)+1}=T^{j(x)}$. In the terminology of Sect. 4, we see that $P(x)$ belongs to the set $S_{j(x)}-S_{j(x)+1}$. These remarks also simplify our task, since we need only compute one of the graded pieces of our data; we obtain the other by applying $\Pi$. Combining these remarks with the previous two lemmas, we obtain the following.

Corollary 42. $\eta^{j(x)}$ is the following set:

$$
\eta^{j(x)}=\{(u, 0) \in \mathbf{N}(G): u \in \mathbf{M}(G) \text { and } \mathbf{F} u=\Pi u\} .
$$

Lemma 43. Define a sublattice $L(x)$ of $\hat{\mathbf{Q}}_{p}^{u r}$.

$$
L(x)=\left\{(a, b):\left|\frac{a x+b}{x-x^{\sigma}}\right| \leqq 1\right\} .
$$


Define a map $v: \mathbf{Q}_{p} \oplus \mathbf{Q}_{p} \rightarrow \mathbf{N}^{j(x)}(G(x))$ by $(a, b) \mapsto(v(a, b), 0)$ where

$$
v(a, b)=\frac{1}{x-x^{\sigma}}\left((a x+b) \gamma^{j(x)}-\left(a x^{\sigma}+b\right) \mathbf{V} \gamma^{j(x)+1}\right) .
$$

Then the lattice $\eta^{j(x)}(G(x))$ is precisely the image of $L(x)$ under $v$.

Proof. Using what we know about $\mathbf{F}$ and $\Pi$, we see that $v=r \gamma^{j(x)}+\mathrm{V} s \gamma^{j(x)+1}$ satisfies $\mathrm{F} u=\Pi u$ if and only if

These relations imply

$$
\begin{aligned}
& r=\alpha r^{\sigma}+\beta r^{\sigma^{2}} \\
& s=\beta r^{\sigma^{2}} .
\end{aligned}
$$

$$
r=\alpha r^{\sigma}+\beta r^{\sigma^{2}} \text {. }
$$

Now we recall that the solutions to this equation are precisely the $\mathbf{Q}_{p}$ span of $1 /\left(x-x^{\sigma}\right)$ and $x /\left(x-x^{\sigma}\right)$ in $\hat{Q}_{p}^{u r}$. (See (6) and the subsequent discussion.) To say that the element $(a, b)$ lies in $L(x)$ is the same as saying that $v(a, b)$ has integral coefficients relative to the basis $\left\{\gamma^{j(x)}, \mathbf{V} \gamma^{j(x)+1}\right\}$ for $\mathbf{M}^{j(x)}$ and therefore that $(v(a, b), 0)$ belongs to $\mathbf{N}(G(x))$.

In the next section we lift all of our data to $W\left(\tilde{\mathbf{F}}_{p}\right)$.

\section{Lifting to $W\left(\overline{\mathbf{F}}_{p}\right)$}

We will use the technique of the universal extension, as described in [Haz, Chap. V, Sect. 30], to lift the point $P(x)$. We learn there that, if $G$ is a formal group of dimension $d$ over $W\left(\overline{\mathbf{F}}_{p}\right)$ with reduction $G_{0}$ over $\overline{\mathbf{F}}_{p}$ of height $h$, there exists an $h$ dimensional formal group $E$ and an exact sequence

$$
0 \rightarrow R \rightarrow E \stackrel{\varepsilon}{\rightarrow} G \rightarrow 0
$$

where $R$ is an additive formal group of dimension $h-d . E$ depends only on $G_{0}$. The Dieudonne module of $E$ is the $\operatorname{Cart}_{p}\left(W\left(\overline{\mathbf{F}}_{p}\right)\right)$ module generated by the symbols $\kappa(g)$ for elements $g \in \mathbf{M}\left(G_{0}\right)$ with the relations $\kappa(\mathbf{F} g)=\mathbf{F} \kappa(g)$ and $\kappa(a g)=a \kappa(g)$ for $a \in W\left(\overline{\mathbf{F}}_{p}\right)$. Furthermore, $\varepsilon(\kappa(g))$ reduces $\bmod p$ to $g$.

Additive subgroups of $E$ classify lifts of $G_{0}$ according to the following theorem.

Theorem 44. (Cartier; see [Haz, Par. 30.3.27]) Lifts $G$ of a fixed commutative formal group $G_{0}$ over $\overline{\mathbf{F}}_{p}$ of dimension d and height $h$ to $W\left(\overline{\mathbf{F}}_{p}\right)$ are classified up to isomorphism by lattices $L \subset \mathbf{V M}\left(G_{0}\right)$ such that $L$ is a free $W\left(\overline{\mathbf{F}}_{p}\right)$ submodule of rank $h-d, M / L$ is free and the map $L / p L \rightarrow(\mathbf{V M}) / p \mathbf{M}$ induced by inclusion is an isomorphism.

We briefly recall how the correspondence of the theorem is realised. Let $R(L)$ be the additive formal group with Lie algebra $L$. The Dieudonne module of $R(L)$ is generated by symbols $\delta(n)$ for $n \in L$ such that $\mathbf{F} \delta(n)=0$ and $\delta(a n)=a \delta(n)$ for $a \in W\left(\overline{\mathbf{F}}_{p}\right)$. Let $\mu: \mathbf{M}(R(L)) \rightarrow \mathbf{M}(E)$ be the unique $\operatorname{Cart}_{p}\left(W\left(\overline{\mathbf{F}}_{p}\right)\right)$-map such that

$$
\mu(\delta(n))=\kappa(n)-\mathbf{V}_{\kappa}\left(\mathbf{V}^{-1} n\right) .
$$


Then the quotient $E / \mu(R(L))$ is a formal group of dimension $d$ which lifts $G_{0}$. Conversely, if $G$ lifts $G_{0}$, let

$$
L=\left\{\boldsymbol{n} \in \mathbf{M}\left(G_{0}\right): \varepsilon(\kappa(n))=0\right\} .
$$

Then $L$ determines $G$ as above.

Since $E$ is a functor of $G_{0}$, if $G_{0}$ is an SFD module, $E$ will inherit the structure of SFD-module. If we wish to lift $G_{0}$ together with its endomorphism structure, we need to specify a lattice $L \subset \mathbf{V M}\left(G_{0}\right)$ which is stable under the action of $\mathscr{O}_{\boldsymbol{D}}$. This means simply that $L$ is a graded submodule, stable under the $\Pi$ action. If these conditions are met, the maps $\varepsilon$ and $\kappa$ will commute with the endomorphism action. We exploit this to obtain our main theorem.

Theorem 45. Let $\left(\psi, G_{0}(x), \rho(x)\right)$ be the point $P(x)$ in $S F D\left(\overline{\mathbf{F}}_{p}\right)$ determined by $\psi \in \operatorname{Hom}\left(\overline{\mathbf{F}}_{p}, \overline{\mathbf{F}}_{p}\right)$ and by $x \in \hat{\mathbf{Q}}_{p}^{\text {ur }}-\mathbf{Q}_{p}$. Let $L(x)$ be the lattice in $\mathbf{M}\left(G_{0}(x)\right)$ spanned by the elements $\mathbf{V} \gamma^{0}(x)$ and $\mathbf{V} \gamma^{1}(x)$, where $\gamma^{0}(x)$ and $\gamma^{1}(x)$ are determined by the matrix $M(x)$ as in equation (12). Let $G(x)$ be the formal group over $W\left(\overline{\mathbf{F}}_{p}\right)$ determined by the lattice $L(x)$ in $\mathbf{M}\left(G_{0}(x)\right)$. Then $z_{0}(\psi, G(x), \rho(x))=x$.

Proof. $L$ is clearly graded and stable under the action of $\Pi$. Further, $L / p L$ is easily seen to be isomorphic to $\mathbf{V M}\left(G_{0}(x)\right) / p \mathbf{M}\left(G_{0}(x)\right)$, and $\mathbf{M}\left(G_{0}(x)\right) / L$ is free of rank 2 . It follows that $G(x)$ exists, so we need only compute the invariants of $G(x)$.

Recall that we have shown (Lemma 40) that $\eta^{j(x)}(G(x))$ consists of all elements $(u, 0) \in \mathbf{N}(G(x))$ such that $\mathbf{F} u=\Pi u$. Drinfeld proves that elements of $\eta\left(G_{0}(x)\right)$ lift uniquely to elements of $\eta\left(G_{0}(x)\right)$. ([Dr, Proposition 2.3]). We have this lift at hand; namely, if $u \in \mathbf{M}\left(G_{0}(x)\right)$ satisfies $\mathbf{F} u=\Pi u$ then $\mathbf{F} \varepsilon \kappa(u)=\Pi \varepsilon \kappa(u)$ since the section $\kappa$ commutes with $\Pi$ and $\mathbf{F}$. We conclude immediately that $\eta^{j(x)}(G(x))=$ $\varepsilon \kappa \eta^{j(x)}\left(G_{0}(x)\right)$.

The tangent space $T$ to $G(x)$ is isomorphic to $\mathbf{M}(G) / \mathbf{V M}(G)$; this in turn is canonically isomorphic to $\mathbf{M}\left(G_{0}\right) / L$. (See [Haz, Chap. V, Sect. 30].) Therefore $T$ is spanned over $W\left(\overline{\mathbf{F}}_{p}\right)$ by the classes of $\varepsilon \kappa\left(\gamma^{0}\right)$ and $\varepsilon \kappa\left(\gamma^{1}\right)$.

In light of the previous remarks about $T$, the map $u: \eta^{j(x)} \rightarrow T^{j(x)}$ is defined by

$$
u\left(\varepsilon \kappa\left(r \gamma^{j(x)}+s \mathbf{V} \gamma^{j(x)+1}\right)\right)=r \varepsilon \kappa\left(\gamma^{j(x)}\right) \quad(\bmod \mathbf{V M}(G(x))) .
$$

Finally, recall that $\rho(x)$ is defined by the matrix $M(x)$ in (12). Applying what we know so far, we see that if $j(x)$ is even we have

$$
\begin{aligned}
u\left(\varepsilon \kappa \rho(x)\left(\left[g^{0}, 0\right]\right)\right) & =u\left(\left[p^{j(x) / 2}\left(x-x^{\sigma}\right)^{-1}\left(x \varepsilon \kappa\left(\gamma^{0}\right)-x^{\sigma} \mathbf{V} \varepsilon \kappa\left(\gamma^{1}\right)\right), 0\right]\right) \\
& =p^{j(x) / 2} \frac{x}{x-x^{\sigma}} \varepsilon \kappa\left(\gamma^{0}\right) \quad(\bmod \mathbf{V M})
\end{aligned}
$$

and a similar calculation tells us that

$$
u\left(\varepsilon \kappa \rho(x)\left(\left[V g^{1}, 0\right]\right)\right)=p^{j(x) / 2} \frac{1}{x-x^{\sigma}} \varepsilon \kappa\left(\gamma^{0}\right) \quad(\bmod V M) .
$$

It follows that in this case, we do indeed have

$$
z_{0}(\psi, G(x), \rho(x))=x \text {. }
$$


A calculation in the case $j(x)$ odd tells us that

$$
\begin{gathered}
u\left(\varepsilon \kappa \rho(x)\left(\left[g^{1}, 0\right]\right)\right)=\frac{p^{(j-1) / 2}}{x-x^{\sigma}} \varepsilon \kappa\left(\gamma^{1}\right) \quad(\bmod \mathbf{V M}) \\
\left.u\left(\varepsilon \kappa \rho(x)\left(\mathbf{V} g^{0}, 0\right]\right)\right)=\frac{p^{(j+1) / 2} x}{x-x^{\sigma}} \varepsilon \kappa\left(\gamma^{1}\right) \quad(\bmod \mathbf{V M}) .
\end{gathered}
$$

This is turn implies that $z_{1}(\psi, G(x), \rho(x))=1 /(p x)$. Using Lemma 31 we see that $z_{0}=1 /\left(p z_{1}\right)=x$, as claimed.

The final section of this paper will give power series for the logarithms of the groups $G(x)$.

\section{Power series}

Our representation of $G(x)$ as a quotient of the universal extension of $G_{0}(x)$ makes it quite simple to describe the logarithm of $G(x)$, thanks to Hazewinkel.

Theorem 46. Let $(\psi, G(x), \rho(x))$ be the point of SFD constructed above. Let $F(x)\left(T_{0}, T_{1}\right)$ be the column vector of power series such that

and

$$
F(x)\left(T_{0}, T_{1}\right)=\left(\begin{array}{c}
T_{0} \\
T_{1}
\end{array}\right)+\cdots
$$

$$
F(x)\left(T_{0}, T_{1}\right)=T+\frac{\alpha(x)}{\Pi} \sigma_{*} F(x)\left(T^{p}\right)+\frac{\beta(x)}{p} \sigma_{*}^{2} F(x)\left(T^{p^{2}}\right)
$$

where we have written

and

$$
T^{p^{n}}=\left(\begin{array}{c}
T_{0}^{p^{n}} \\
T_{1}^{p^{n}}
\end{array}\right)
$$

$$
\Pi=\left(\begin{array}{ll}
0 & p \\
1 & 0
\end{array}\right)
$$

Then $F(x)$ is the logarithm of $G(x)$. In other words,

$$
G(x)(X ; Y)=F(x)^{-1}(F(x)(X)+F(x)(Y))
$$

defines a formal group law over $W\left(\overline{\mathbf{F}}_{p}\right)$ isomorphic to $G(x)$. Fix an action of $\mathcal{O}_{D}$ on this formal group so that $\Pi$ acts via the above matrix (i.e. as $F^{-1}(\Pi F)$ ) and so that $a \in A$ acts as $F^{-1}([a] F)$ where

$$
[a]=\left(\begin{array}{cc}
\psi \sigma_{j(x)}(a) & 0 \\
0 & \psi \sigma_{j(x)+1}(a)
\end{array}\right)
$$

where we recall that the $\sigma_{i}$ are the two labelled embeddings of $A \rightarrow W\left(\overline{\mathbf{F}}_{p}\right)$. The resulting $S F D$ module $G(x)(X, Y)$ is isomorphic as $S F D$ module to $G(x)$.

Proof. All of this is simply a translation of the statements about Dieudonne modules we have made before. The necessary integrality results follow from the 
functional equation lemma of [Haz, Par. 10.2]. The recursion relation for $F$ follows from [Haz, Par. 30.2.32] and Lemma 15. The action of $\Pi$ then follows from (13). The action of $A \subset \mathcal{O}_{D}$ comes from the definition of the grading and the action of $A$ on $\psi_{*} \Phi$.

Let us remark that if $\tau$ is in $S L_{2}\left(\mathbf{Q}_{p}\right)$, we must have $G_{\tau x}$ isomorphic to $G_{x}$. The modular properties of $\alpha$ and $\beta$ make this isomorphism immediate if we substitue $\tau x$ into the recursion relation in (15) and see what happens. We leave this to the reader.

\section{Conclusion}

All of our results are stated for 2-dimensional modules with action by the quaternion division algebra over $\mathbf{Q}_{p}$. Drinfeld's results are true in much greater generality. We remark that our results generalize easily to the case of 2-dimensional modules with action by quaternion algebras over extensions of $\mathbf{Q}_{p}$ by applying the theory of formal $\mathcal{O}$-modules. We suspect that Drinfeld's higher dimensional modules over $W\left(\overline{\mathbf{F}}_{p}\right)$ are determined by formulae similar to that in Theorem 46 , but with $\alpha$ and $\beta$ generalized to moduli for higher dimensional $\mathbf{Q}_{p}$-subspaces of $\hat{\mathbf{Q}}_{p}^{\text {ur }}$; however, we have not investigated this in any detail.

Although this work amplifies Drinfeld's original paper by supplying many details in certain cases, it is seriously limited in that it considers lifts of SFD modules to unramified rings only. The most interesting points in the $p$-adic upper half plane are the points defined over ramified rings, which reduce $\bmod p$ to the singular points on the special fiber. What happens there? We do not have a simple answer.

Drinfeld's moduli for formal groups on the $p$-adic upper half plane is the basis for his proof that Shimura curves have $p$-adic uniformizations. In a later work, we hope to exploit improved versions of the techniques in this work to better understand the arithmetic of Shimura curves. In particular, in the course of work on $p$-adic $L$ functions, we have been led to construct certain " $p$-adic periods" associated to the cohomology of sheaves on Shimura curves which depend essentially on the existence of a $p$-adic uniformization. We hope to use Drinfeld's moduli to obtain a more natural construction of these periods in terms of the Gauss-Manin connection, and thereby to gain a better understanding of how they might come to appear in special values of $p$-adic $L$-functions.

\section{References}

[B] Bosch, S.: Zur Kohomologietheorie Rigid Analytischer Räume. Manuscr. Math. 20, 1-27 (1977)

[Dr] Drinfeld, V.G.: Coverings of p-adic symmetric regions. Funct. Anal. Appl. 10, 29-40 (1976)

[Haz] Hazewinkel, M.: Formal groups and applications. New York: Academic Press 1978

[Man] Manin, Yu.: p-adic automorphic functions. J. Sov. Math. 279-334 (1976)

[Mum] Mumford, D.: An analytic construction of degenerating curves over complete local rings. Compos. Math, 24, 129-174 (1972)

Received December 22, 1988 